\title{
Existence and Relaxation Results for Second Order Multivalued Systems
}

\section{Nikolaos S. Papageorgiou ${ }^{1} \cdot$ Calogero Vetro $^{2}$}

Received: 25 January 2021 / Accepted: 23 April 2021 / Published online: 4 May 2021

(c) The Author(s) 2021

\begin{abstract}
We consider nonlinear systems driven by a general nonhomogeneous differential operator with various types of boundary conditions and with a reaction in which we have the combined effects of a maximal monotone term $A(x)$ and of a multivalued perturbation $F(t, x, y)$ which can be convex or nonconvex valued. We consider the cases where $D(A) \neq \mathbb{R}^{N}$ and $D(A)=\mathbb{R}^{N}$ and prove existence and relaxation theorems. Applications to differential variational inequalities and control systems are discussed.
\end{abstract}

Mathematics Subject Classification (2010) 34B15 · 34C25 · 47H06

Keywords Maximal monotone map · Convex and nonconvex problems · Continuous and measurable selections $\cdot$ Relaxation $\cdot$ Optimal control

\section{Introduction}

In this paper, we study the following second order multivalued boundary value problem

$$
\left\{\begin{array}{l}
a\left(u^{\prime}(t)\right)^{\prime} \in A(u(t))+F\left(t, u(t), u^{\prime}(t)\right) \text { for a.a. } t \in T=[0, b], \\
u \in B C .
\end{array}\right.
$$

In this problem $B C$ stands for one of the following boundary conditions:

- $u(0)=u(b)=0$ (Dirichlet problem),

- $u^{\prime}(0)=u^{\prime}(b)=0$ (Neumann problem),

- $u(0)=u(b), u^{\prime}(0)=u^{\prime}(b)$ (periodic problem),

- $u^{\prime}(0)=u(b)=0$ (mixed problem $)$.

\section{Vetro}

calogero.vetro@unipa.it

N.S. Papageorgiou

npapg@math.ntua.gr

1 Department of Mathematics, National Technical University, Zografou campus, 15780, Athens, Greece

2 Department of Mathematics and Computer Science, University of Palermo, Via Archirafi 34, 90123, Palermo, Italy 
Also in the differential operator (left hand side of (1)), the map $a: \mathbb{R}^{N} \rightarrow \mathbb{R}^{N}$ is continuous, strictly monotone and in general not homogeneous. Moreover, we do not require that $a(\cdot)$ satisfies a polynomial growth condition. So, the differential operator in (1) is very general and incorporates as special cases many well-known differential operators that we encounter in the literature. In the right hand side (reaction) of the problem we have two terms. One is the map $A: D(A) \subseteq \mathbb{R}^{N} \rightarrow 2^{\mathbb{R}^{N}}$ which is maximal monotone. We do not require that $D(A)=\mathbb{R}^{N}$ and so we incorporate in our framework systems with unilateral constraints (differential variational inequalities). The perturbation $F\left(t, u, u^{\prime}\right)$ is multivalued. We consider the cases where $F$ has convex values (convex problem) and nonconvex values (nonconvex problem). We prove existence theorems for both cases. Then we ask the question of whether the solutions of the convex problem can be approximated by solutions of the nonconvex one. Such a result is known in the literature as "relaxation theorem" and it has important consequences in many applied areas. For example, in the context of control systems, it implies that we can economize in the use of control functions. For the question of relaxation, we have only a partial answer. Namely we show the result only for Dirichlet problems, under more restrictive conditions on the map $a(\cdot)$ and with the perturbation $F$ being independent of $u^{\prime}$. When $\operatorname{dom} A=\mathbb{R}^{N}$, some of these restrictions on $a(\cdot)$ and $F$ can be removed, but again we have to restrict ourselves to the Dirichlet problem. In the last section, we present applications of our existence results. One concerns differential variational inequalities and the others control and optimal control problems with second order dynamics.

Our approach is topological based on a multivalued version of the Leray-Schauder principle (see Papageorgiou-Rădulescu-Repovš [22], Proposition 3.2.22, p. 198), due to Bader [1]. In the literature such problems are usually approached by using some variant of the Hartman or Nagumo-Hartman condition, which leads to an a priori uniform bound for the solutions. We refer to the works of Frigon-Montoki [4], Halidias-Papageorgiou [10], Kandilakis-Papageorgiou [12], Kyritsi-Matzakos-Papageorgiou [13], Ma-Xue [15], Pruszko [25], Zhang-Li [26] and the recent work of Gasiński-Papageorgiou [9] for first order systems. Here instead we employ a condition which involves the principal eigenvalue of the corresponding eigenvalue problem for the vector $p$-Laplacian. A similar condition can be found in the work of Papageorgiou-Vetro-Vetro [21] for multivalued Duffing systems with no maximal monotone term (that is, $A \equiv 0$ ) and with Dirichlet boundary condition. In the next section we briefly recall the main mathematical tools which we will need in the analysis of the problem and also state the hypotheses on the data of (1).

\section{Mathematical Background - Hypotheses}

We will use tools from multivalued analysis (see Hu-Papageorgiou [11]) and from the theory of nonlinear operators of monotone type (see Gasiński-Papageorgiou [7]).

Let $X$ be a Banach space. We will use the following notation:

$$
\begin{aligned}
P_{f(c)}(X) & =\{B \subseteq X: B \text { is nonempty, closed (and convex) }\} \\
P_{(w) k(c)}(X) & =\{B \subseteq X: B \text { is nonempty, (weakly-) compact (and convex) }\} .
\end{aligned}
$$

Let $(\Omega, \Sigma)$ be a measurable space and assume that $X$ is a separable Banach space. Given a multifunction $F: \Omega \rightarrow 2^{X} \backslash\{\emptyset\}$, we say that $F(\cdot)$ is "graph measurable", if

$$
\operatorname{Gr} F=\{(\omega, x) \in \Omega \times X: x \in F(\omega)\} \in \Sigma \otimes B(X),
$$


with $B(X)$ being the Borel $\sigma$-field of $X$. If $\Sigma=\widehat{\Sigma}=$ the universal $\sigma$-field (this is true if there is a $\sigma$-finite measure $\mu$ on $\Sigma$, with $\Sigma$ being $\mu$-complete), then a graph measurable multifunction $F: \Omega \rightarrow 2^{X} \backslash\{\emptyset\}$ admits a measurable selection, that is, there exists a $(\Sigma, B(X))$-measurable function $f: \Omega \rightarrow X$ such that $f(\omega) \in F(\omega)$ for all $\omega \in \Omega$. This is the celebrated Yankov-von Neumann-Aumann selection theorem (see Hu-Papageorgiou [11], p. 158). In fact we can find a whole sequence $\left\{f_{n}\right\}_{n \in \mathbb{N}}$ of such measurable selections such that

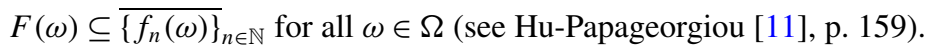

A multifunction $F: \Omega \rightarrow P_{f}(X)$ is said to be "measurable", if for all $u \in X$, the function $\omega \rightarrow d(u, F(\omega))=\inf \left[\|u-x\|_{X}: x \in F(\omega)\right]$ is $\Sigma$-measurable. A measurable multifunction $F: \Omega \rightarrow P_{f}(X)$ is graph measurable and the converse is true for $P_{f}(X)$-valued multifunctions, if there is a $\sigma$-finite, complete measure $\mu$ defined on $\Sigma$.

Let $(\Omega, \Sigma, \mu)$ be a $\sigma$-finite measure space and $X$ a separable Banach space. For any $1 \leq p \leq \infty$ and for any multifunction $F: \Omega \rightarrow 2^{X} \backslash\{\emptyset\}$, we define the set

$$
S_{F}^{p}=\left\{f \in L^{p}(\Omega, X): f(\omega) \in F(\omega) \quad \mu \text {-a.e. }\right\} .
$$

If $F(\cdot)$ is graph measurable and $\omega \rightarrow \inf \left\{\|x\|_{X}: x \in F(\omega)\right\}$ belongs in $L^{p}(\Omega)$, then $S_{F}^{p} \neq$ $\emptyset$. This set is decomposable in the sense that for all triples $\left(B, f_{1}, f_{2}\right) \in \Sigma \times S_{F}^{p} \times S_{F}^{p}$, we have

$$
\chi_{B} f_{1}+\chi_{B^{c}} f_{2} \in S_{F}^{p} \quad\left(B^{c}=\Omega \backslash B\right) .
$$

Here by $\chi_{B}$ we denote the characteristic function for the set $B \in \Sigma$, that is,

$$
\chi_{B}(\omega)= \begin{cases}1 & \text { if } \omega \in B, \\ 0 & \text { if } \omega \notin B .\end{cases}
$$

Since $\chi_{B^{c}}=1-\chi_{B}$, the above definition of decomposability formally looks like that of convexity. Only now the coefficients are not constants in $[0,1]$, but functions with values in $[0,1]$. Nevertheless decomposable sets exhibit properties similar to those of convex sets (see Fryszkowski [6] and Hu-Papageorgiou [11]).

Suppose that $Y, V$ are Hausdorff topological spaces and $G: Y \rightarrow 2^{V} \backslash\{\emptyset\}$ a multifunction. We introduce the following notions:

(a) We say that $G(\cdot)$ is "upper semicontinuous" ("usc" for short), if for all $U \subseteq V$ open, the set $G^{+}(U)=\{y \in Y: G(y) \subseteq U\}$ is open.

(b) We say that $G(\cdot)$ is "lower semicontinuous" ("lsc" for short), if for all $U \subseteq V$ open, the set $G^{-}(U)=\{y \in Y: G(y) \cap U \neq \emptyset\}$ is open.

(c) We say that $G(\cdot)$ is "closed", if $\operatorname{Gr} G=\{(y, v) \in Y \times V: v \in G(y)\} \subseteq Y \times V$ is closed.

When $F$ is single valued, then both notions in (a) and (b) coincide with that of continuity. Upper semicontinuity implies closedness, while the converse is true if $G(\cdot)$ is locally compact, that is, for every $y \in Y$, there is a neighborhood $W$ of $y$ such that $\underset{y^{\prime} \in W}{\cup} G\left(y^{\prime}\right) \in P_{k}(V)$. If $V$ is a metric space with metric $d_{V}$, then $G(\cdot)$ is lsc if and only if for every $v \in V$ the function $y \rightarrow d_{V}(v, G(y))$ is upper semicontinuous as an $\mathbb{R}_{+}$-valued function.

For a metric space $V$ with metric $d_{V}$, on $P_{f}(V)$ we can define a generalized metric, known as the "Hausdorff metric" by

$$
h(B, C)=\sup \left[\left|d_{V}(v, B)-d_{V}(v, C)\right|: v \in V\right] \quad \text { for all } B, C \in P_{f}(V) .
$$


We know that if $V$ is complete, then so is $\left(P_{f}(V), h\right)$. A multifunction $G: Y \rightarrow P_{f}(V)$ is said to be " $h$-continuous", if it is continuous from $Y$ into $\left(P_{f}(V), h\right)$. We say that $G(\cdot)$ is "continuous", if it is both usc and lsc. The two notions are in general distinct and they coincide if $G(\cdot)$ is $P_{k}(V)$-valued.

For a Banach space $X$ and $C \subseteq X$ nonempty, we define

$$
|C|=\sup \left[\|u\|_{X}: u \in C\right] .
$$

Let $Y, V$ be two Banach spaces and $\xi: Y \rightarrow V$. We say that $\xi(\cdot)$ is "completely continuous", if $y_{n} \stackrel{w}{\rightarrow} y$ in $Y$ implies $\xi\left(y_{n}\right) \rightarrow \xi(y)$ in $V$ (so it is sequentially continuous from $Y$ with the weak topology into $V$ with the strong topology). A multifunction $G: Y \rightarrow 2^{V} \backslash\{\emptyset\}$ is "compact", if it is usc and maps bounded sets in $Y$ into relatively compact sets in $V$.

We will use two results from multivalued analysis. The first is the multivalued analog of the Leray-Schauder Alternative Principle due to Bader [1]. So, assume that $Y, V$ are Banach spaces, $N: Y \rightarrow P_{w k c}(V)$ is usc from $Y$ into $V_{w}=$ the Banach space $V$ endowed with the weak topology and $\xi: V \rightarrow Y$ is completely continuous. Let $L=\xi \circ N$. The result of Bader [1] (Theorem 8) asserts the following:

Theorem 1 If $Y, V, L$ are as above and $L(\cdot)$ is compact, then one of the following statements holds:

(a) $S=\{y \in Y: y \in \mu L(y), 0<\mu<1\}$ is unbounded; or

(b) $L(\cdot)$ admits a fixed point (that is, there exists $y_{0} \in Y$ such that $y_{0} \in L\left(y_{0}\right)$ ).

The next theorem is an extension of the celebrated Michael Selection Theorem (see [11], p. 92) to multifunctions with decomposable values. The result is a powerful illustration that decomposability is a good substitute for convexity and it is due to Bressan-Colombo [2] and Fryszkowski [5].

Theorem 2 If $(\Omega, \Sigma, \mu)$ is a finite measure space, $X$ is a separable Banach space, $Y$ is a separable metric space and $N: Y \rightarrow P_{f}\left(L^{1}(\Omega, X)\right)$ is a lsc multifunction with decomposable values, then there exists a continuous map $e: Y \rightarrow L^{1}(\Omega, X)$ such that

$$
e(y) \in N(y) \text { for all } y \in Y \text {. }
$$

Now let $E$ be a reflexive Banach space and $E^{*}$ its topological dual. By $\langle\cdot, \cdot\rangle$ we denote the duality brackets for the pair $\left(E, E^{*}\right)$. A map $A: E \rightarrow 2^{E^{*}}$ is said to be "monotone", if

$$
\left\langle u^{*}-v^{*}, u-v\right\rangle \geq 0 \quad \text { for all }\left(u, u^{*}\right),\left(v, v^{*}\right) \in \operatorname{Gr} A .
$$

Recall that $\operatorname{Gr} A=\left\{\left(u, u^{*}\right) \in E \times E^{*}: u^{*} \in A(u)\right\}$. We say that $A(\cdot)$ is "strictly monotone", if

$$
\left\langle u^{*}-v^{*}, u-v\right\rangle>0 \quad \text { for all }\left(u, u^{*}\right),\left(v, v^{*}\right) \in \operatorname{Gr} A, u \neq v .
$$

We say that $A(\cdot)$ is "maximal monotone", if Gr $A$ is not properly included in the graph of another monotone map, that is,

$$
\left\langle u^{*}-v^{*}, u-v\right\rangle \geq 0 \quad \text { for all }\left(u, u^{*}\right) \in \operatorname{Gr} A \quad \Longrightarrow \quad\left(v, v^{*}\right) \in \operatorname{Gr} A .
$$


If $A(\cdot)$ is maximal monotone, then $\mathrm{Gr} A$ is closed in $E \times E_{w}^{*}$ and in $E_{w} \times E^{*}$. Here by $E_{w}$ (resp. by $E_{w}^{*}$ ), we denote the space $E$ (resp. $E^{*}$ ) equipped with the weak topology. Also we set $D(A)=\{u \in E: A(u) \neq \emptyset\}$ (the domain of $A$ ).

Suppose that $E=H=$ a Hilbert space and identify $H$ with its dual (that is, $H=H^{*}$, by the Riesz-Frechet Theorem). Let $A: H \rightarrow 2^{H}$ be a maximal monotone map. We introduce the following single-valued maps:

$$
\begin{array}{r}
J_{\lambda}=(\mathrm{id}+\lambda A)^{-1} \quad(\text { the resolvent of } A), \\
A_{\lambda}=\frac{1}{\lambda}\left[\mathrm{id}-J_{\lambda}\right] \quad(\text { the Yosida approximation of } A) \\
\quad(\lambda>0) .
\end{array}
$$

Proposition 1 If $A: H \rightarrow 2^{H}$ is maximal monotone and $\lambda>0$, then

(a) $J_{\lambda}: H \rightarrow H$ is nonexpansive, that is,

$$
\left\|J_{\lambda}(u)-J_{\lambda}(v)\right\|_{H} \leq\|u-v\|_{H} \quad \text { for all } u, v \in H
$$

(b) $A_{\lambda}(u) \in A\left(J_{\lambda}(u)\right)$ for all $u \in H$;

(c) $A_{\lambda}(\cdot)$ is monotone and $\frac{1}{\lambda}$-Lipschitz continuous, that is,

$$
\left\|A_{\lambda}(u)-A_{\lambda}(v)\right\|_{H} \leq \frac{1}{\lambda}\|u-v\|_{H} \quad \text { for all } u, v \in H
$$

(d) $\left\|A_{\lambda}(u)\right\|_{H} \leq\left\|A^{0}(u)\right\|=\min \left[\left\|u^{*}\right\|_{H}: u^{*} \in A(u)\right]$ and $A_{\lambda}(u) \rightarrow A^{0}(u)$ in $H$ as $\lambda \rightarrow 0^{+}$ for all $u \in D(A)$

(e) $\overline{D(A)}$ is convex and $J_{\lambda}(u) \rightarrow \operatorname{proj}(u, \overline{D(A)})$ as $\lambda \rightarrow 0^{+}$for all $u \in H$.

Remark 1 We know that in a Hilbert space every closed, convex set has the best approximation property. So, in part (e) of the above proposition, the metric projection $\operatorname{proj}(u, \overline{D(A)})$ is well-defined.

We will also use an extension of the notion of maximal monotone operator. So, as before $E$ is a reflexive Banach space with $E^{*}$ its topological dual. A multivalued map $L: E \rightarrow$ $P_{w k c}\left(E^{*}\right)$ is said to be "pseudomonotone" if it maps bounded sets to bounded sets and has the following property

$$
\begin{gathered}
\text { "for every sequences }\left\{u_{n}\right\}_{n \in \mathbb{N}} \subseteq E,\left\{u_{n}^{*}\right\}_{n \in \mathbb{N}} \subseteq E^{*} \text {, such that } \\
u_{n} \stackrel{w}{\rightarrow} u \text { in } E, u_{n}^{*} \stackrel{w}{\rightarrow} u^{*} \text { in } E^{*}, \limsup _{n \rightarrow+\infty}\left\langle u_{n}^{*}, u_{n}-u\right\rangle \leq 0 \\
\text { we have } \\
\left(u, u^{*}\right) \in \operatorname{Gr} L \text { and }\left\langle u_{n}^{*}, u_{n}\right\rangle \rightarrow\left\langle u^{*}, u\right\rangle . "
\end{gathered}
$$

If $L(\cdot)$ is maximal monotone and $D(L)=E$, then $L(\cdot)$ is pseudomonotone (for details we refer to Gasiński-Papageorgiou [7], Sect. 3.2).

Finally we mention that by $\|\cdot\|$ we denote the norm of the Sobolev space $W^{1, p}(\Omega)$. Recall that

$$
\|u\|=\left[\|u\|_{p}^{p}+\left\|u^{\prime}\right\|_{p}^{p}\right]^{1 / p} \quad \text { for all } u \in W^{1, p}(\Omega) .
$$

Now, we are ready to introduce the hypotheses on the data of problem (1). First for the map $a(\cdot)$ in the differential operator. 
$H_{0}: a: \mathbb{R}^{N} \rightarrow \mathbb{R}^{N}$ has the form $a(y)=a_{0}(|y|) y$ for all $y \in \mathbb{R}^{N}$, with $a_{0}(t)>0$ for all $t>0$ and

(i) $a(\cdot)$ is continuous, strictly monotone;

(ii) there exists $c_{0}>0$ such that

$$
c_{0}|y|^{p} \leq(a(y), y)_{\mathbb{R}^{N}} \quad \text { for all } y \in \mathbb{R}^{N}, 2 \leq p .
$$

Remark 2 Hypothesis $H_{0}(\mathrm{i})$ implies that $a(\cdot)$ is maximal monotone. It is worth pointing out that no global growth condition is imposed on $a(\cdot)$. So, the map is very general. Hypotheses $H_{0}$ imply that $a: \mathbb{R}^{N} \rightarrow \mathbb{R}^{N}$ is a homeomorphism and we have $\left|a^{-1}(y)\right| \rightarrow+\infty$ as $|y| \rightarrow+\infty$. The restriction $2 \leq p$ is needed since we will not assume that $D(A)=\mathbb{R}^{N}$. When $D(A)=\mathbb{R}^{N}$, then we can have $1<p$. Such kind of maps $a(\cdot)$, were first used by Manásevich-Mawhin [16].

Example 1 The following maps satisfy hypotheses $H_{0}$ :

(a) $a(y)=|y|^{p-2} y, 2 \leq p<+\infty$.

This map corresponds to the vector $p$-Laplacian.

(b) $a(y)=|y|^{p-2} y+|y|^{q-2} y, 1<q<p<+\infty, 2 \leq p<+\infty$.

This map corresponds to the $(p, q)$-Laplacian.

(c) $a(y)=\left[1+|y|^{2}\right]^{\frac{p-2}{2}} y, 2 \leq p<+\infty$.

(d) $a(y)=|y|^{p-2} y\left[1+e^{|y|^{p}}\right], 2 \leq p<+\infty$.

$H_{1}: A: \mathbb{R}^{N} \rightarrow 2^{\mathbb{R}^{N}}$ is a maximal monotone map.

Remark 3 We stress that we do not assume that $D(A)=\mathbb{R}^{N}$. This way our setting covers problems with unilateral constraints. Also this fact leads to the restriction $2 \leq p$.

In what follows by $\widehat{\lambda}_{1}$ we denote the principal eigenvalue of the eigenvalue problem

$$
-\left(\left|u^{\prime}\right|^{p-2} u^{\prime}\right)^{\prime}=\widehat{\lambda}|u|^{p-2} u, \quad u \in B C .
$$

These eigenvalue problems are discussed in Manásevich-Mawhin [17] and we have

$$
\begin{array}{ll}
\widehat{\lambda}_{1}>0 & \text { for the Dirichlet and mixed problems, } \\
\widehat{\lambda}_{1}=0 & \text { for the Neumann and periodic problems. }
\end{array}
$$

For the convex problem our hypotheses on the multivalued perturbation $F(t, x, y)$ are the following:

$H_{2}: F: T \times \mathbb{R}^{N} \times \mathbb{R}^{N} \rightarrow P_{k c}\left(\mathbb{R}^{N}\right)$ is a multifunction such that

(i) for every $x, y \in \mathbb{R}^{N}, t \rightarrow F(t, x, y)$ is graph measurable;

(ii) for a.a. $t \in T,(x, y) \rightarrow F(t, x, y)$ is closed;

(iii) if $\sigma(t, x, y)=\inf \left[(v, x)_{\mathbb{R}^{N}}: v \in F(t, x, y)\right]$, then

$$
\liminf _{|x| \rightarrow+\infty} \frac{\sigma(t, x, y)}{|x|^{p}} \geq \vartheta(t) \quad \text { uniformly for a.a. } t \in T \text {, all } y \in \mathbb{R}^{N} \text {, }
$$

with $\vartheta \in L^{\infty}(T), \vartheta(t) \geq-c_{0} \widehat{\lambda}_{1}$ for a.a. $t \in T, \vartheta \not \equiv-c_{0} \widehat{\lambda}_{1}$; 
(iv) $|F(t, x, y)| \leq \eta_{1}(t,|x|)+\eta_{2}(t,|x|)|y|^{p-1}$ for a.a. $t \in T$, all $x, y \in \mathbb{R}^{N}$ and

$$
\begin{aligned}
& \sup \left[\eta_{1}(t, r): 0 \leq r \leq M\right] \leq \gamma_{1, M}(t) \text { for a.a. } t \in T, \text { with } \gamma_{1, M} \in L^{2}(T), \\
& \sup \left[\eta_{2}(t, r): 0 \leq r \leq M\right] \leq \gamma_{2, M}(t) \text { for a.a. } t \in T, \text { with } \gamma_{2, M} \in L^{\infty}(T) .
\end{aligned}
$$

For the nonconvex problem, the hypotheses on the multivalued perturbation are the following:

$H_{3}: F: T \times \mathbb{R}^{N} \times \mathbb{R}^{N} \rightarrow P_{f}\left(\mathbb{R}^{N}\right)$ is a multifunction such that

(i) $\operatorname{Gr} F \in \mathcal{L}_{T} \otimes B\left(\mathbb{R}^{N}\right) \otimes B\left(\mathbb{R}^{N}\right)$ with $\mathcal{L}_{T}$ being the Lebesgue $\sigma$-algebra of $T$ and $B\left(\mathbb{R}^{N}\right)$ is the Borel $\sigma$-algebra of $\mathbb{R}^{N}$;

(ii) for a.a. $t \in T,(x, y) \rightarrow F(t, x, y)$ is lsc;

(iii) same as hypothesis $H_{1}$ (iii);

(iv) same as hypothesis $H_{1}$ (iv).

Remark 4 Now the measurability hypothesis (see $H_{2}(\mathrm{i})$ ) is stronger. This is always the case for nonconvex problems.

\section{Convex Problem}

In this section we consider the case when $F$ is convex-valued.

Setting $\widehat{F}(t, x, y)=F(t, x, y)-|x|^{p-2} x$, for every $\lambda>0$, we consider the following auxiliary boundary value problem

$$
\left\{\begin{array}{l}
a\left(u^{\prime}(t)\right)^{\prime}-|u(t)|^{p-2} u(t) \in A_{\lambda}(u(t))+\widehat{F}\left(t, u(t), u^{\prime}(t)\right) \quad \text { a.e. on } T, \\
u \in B C .
\end{array}\right.
$$

We will solve $\left(2_{\lambda}\right)$. In order to simplify the notation, we write $L^{r}=L^{r}\left(T, \mathbb{R}^{N}\right), W^{1, r}=$ $W^{1, r}\left((0, b), \mathbb{R}^{N}\right)$ for all $1 \leq r \leq+\infty$ and $C^{1}=C^{1}\left(T, \mathbb{R}^{N}\right)$. We consider the map $\widehat{a}: D(\widehat{a}) \subseteq$ $L^{p} \rightarrow L^{p^{\prime}}\left(\frac{1}{p}+\frac{1}{p^{\prime}}=1\right)$ defined by

$$
\widehat{a}(u)(\cdot)=-a\left(u^{\prime}(\cdot)\right)^{\prime}
$$

for all $u \in D(\widehat{a})=\left\{y \in C^{1}: a\left(y^{\prime}(\cdot)\right) \in W^{1, p^{\prime}}, y \in B C\right\}$. From Kyritsi-Matzakos-Papageorgiou [13] (Proposition 3), we have the following result concerning this map (the same proof is valid for all four boundary conditions).

Proposition 2 If hypotheses $H_{0}$ hold, then the map $\widehat{a}: D(\widehat{a}) \subseteq L^{p} \rightarrow L^{p^{\prime}}$ is maximal monotone.

Let $\widehat{A_{\lambda}}: L^{p} \rightarrow L^{p^{\prime}}$ be defined by

$$
\widehat{A_{\lambda}}(u)(\cdot)=A_{\lambda}(u(\cdot)) \quad \text { for all } u \in L^{p} .
$$

Since $A_{\lambda}(\cdot)$ is Lipschitz continuous, $A_{\lambda}(0)=0$ and $2 \leq p$, we see that $\widehat{A_{\lambda}}$ is well-defined and we have:

Proposition 3 If hypotheses $H_{1}$ hold and $\lambda>0$, then the map $\widehat{A}_{\lambda}: L^{p} \rightarrow L^{p^{\prime}}$ is monotone, continuous (hence maximal monotone). 
Remark 5 It is at this point that we use the restriction $2 \leq p$. If $D(A)=\mathbb{R}^{N}$, then we do not need to consider the approximate boundary value problem $\left(2_{\lambda}\right)$ and so we do not need the restriction $2 \leq p$.

Given $h \in L^{p^{\prime}}$, we consider the following boundary value problem

$$
\left\{\begin{array}{l}
a\left(u^{\prime}(t)\right)^{\prime}-|u(t)|^{p-2} u(t) \in A_{\lambda}(u(t))+h(t) \quad \text { a.e. on } T, \\
u \in B C .
\end{array}\right.
$$

Proposition 4 If hypotheses $H_{0}, H_{1}$ hold and $\lambda>0$, then problem $\left(3_{\lambda}\right)$ admits a unique solution $\widehat{u}_{\lambda} \in C^{1}$.

Proof Let $\varphi_{p}: L^{p} \rightarrow L^{p^{\prime}}$ be the map defined by

$$
\varphi_{p}(u)(\cdot)=|u(\cdot)|^{p-2} u(\cdot) \quad \text { for all } u \in L^{p} .
$$

Consider the map $V_{\lambda}: D\left(V_{\lambda}\right) \subseteq L^{p} \rightarrow L^{p^{\prime}}$ defined by

$$
V_{\lambda}(u)=\widehat{a}(u)+\varphi_{p}(u)+\widehat{A}_{\lambda}(u) \text { for all } u \in D(\widehat{a}) .
$$

Evidently $D\left(V_{\lambda}\right)=D(\widehat{a})$ and from Theorem 3.2.41, p. 328, of Gasiński-Papageorgiou [7], we have that $V_{\lambda}(\cdot)$ is maximal monotone. Let $(\cdot, \cdot)_{p p^{\prime}}$ denote the duality brackets for the pair $\left(L^{p}, L^{p^{\prime}}\right)$. We have

$$
\begin{aligned}
& \left(V_{\lambda}(u), u\right)_{p p^{\prime}} \\
= & \int_{0}^{b}\left(-a\left(u^{\prime}\right)^{\prime}, u\right)_{\mathbb{R}^{N}} d t+\|u\|_{p}^{p}+\int_{0}^{b}\left(A_{\lambda}(u), u\right)_{\mathbb{R}^{N}} d t \\
\geq & \int_{0}^{b}\left(a\left(u^{\prime}\right), u^{\prime}\right)_{\mathbb{R}^{N}} d t+\|u\|_{p}^{p}
\end{aligned}
$$

(performing integration by parts and since $A_{\lambda}(0)=0$ )

$\geq c_{0}\left\|u^{\prime}\right\|_{p}^{p}+\|u\|_{p}^{p} \quad\left(\right.$ see hypothesis $\left.H_{0}(\mathrm{ii})\right)$,

$\Rightarrow \quad V_{\lambda}(\cdot)$ is coercive.

Then Corollary 3.2.31, p. 319, of Gasiński-Papageorgiou [7] implies that

$$
V_{\lambda}(\cdot) \text { is surjective. }
$$

So, we can find $\widehat{u}_{\lambda} \in D(\widehat{a}) \subseteq C^{1}$ such that

$$
V_{\lambda}\left(\widehat{u}_{\lambda}\right)=h
$$

In fact, on account of the strict monotonicity of $\varphi_{p}(\cdot)$ this solution is unique.

In what follows $X$ stands for one of the following spaces for vector-valued functions:

$$
\begin{aligned}
& X=C_{0}^{1} \quad \text { for the Dirichlet problem } \\
& \qquad C_{0}^{1}=\left\{u \in C^{1}\left(T, \mathbb{R}^{N}\right): u(0)=u(b)=0\right\},
\end{aligned}
$$


$X=C^{1}$ for the Neumann problem,

$X=C_{\mathrm{per}}^{1} \quad$ for the periodic problem

$$
C_{\mathrm{per}}^{1}=\left\{u \in C^{1}\left(T, \mathbb{R}^{N}\right): u(0)=u(b), u^{\prime}(0)=u^{\prime}(b)\right\},
$$

$X=C_{*}^{1} \quad$ for the mixed problem

$$
C_{*}^{1}=\left\{u \in C^{1}\left(T, \mathbb{R}^{N}\right): u^{\prime}(0)=u(b)=0\right\} .
$$

Consider the map $\xi_{\lambda}: L^{p^{\prime}} \rightarrow D\left(V_{\lambda}\right)=D(\widehat{a}) \subseteq X$ defined by

$$
\xi_{\lambda}(h)=\widehat{u}_{\lambda}
$$

with $\widehat{u}_{\lambda}$ being the unique solution of $\left(3_{\lambda}\right)$ guaranteed by Proposition 4 .

Proposition 5 If hypotheses $H_{0}, H_{1}$ hold and $\lambda>0$, then the solution map $\xi_{\lambda}: L^{p^{\prime}} \rightarrow X$ is completely continuous.

Proof Consider a sequence $h_{n} \stackrel{w}{\rightarrow} h$ in $L^{p^{\prime}}$ and let $\widehat{u}_{n}=\xi_{\lambda}\left(h_{n}\right), n \in \mathbb{N}$, and $\widehat{u}=\xi_{\lambda}(h)$. We have

$$
\begin{aligned}
& \widehat{a}\left(\widehat{u}_{n}\right)+\varphi_{p}\left(\widehat{u}_{n}\right)+\widehat{A}_{\lambda}\left(\widehat{u}_{n}\right)=h_{n} \quad \text { in } L^{p^{\prime}} \text { for all } n \in \mathbb{N}, \\
\Rightarrow \quad & c_{0}\left\|\widehat{u}_{n}^{\prime}\right\|_{p}^{p}+\left\|\widehat{u}_{n}\right\|_{p}^{p} \leq c_{1}\left\|\widehat{u}_{n}\right\| \quad \text { for some } c_{1}>0, \text { all } n \in \mathbb{N} \\
& \text { (acting with } \widehat{u}_{n} \text { and performing integration by parts), } \\
\Rightarrow \quad & \left\{\widehat{u}_{n}\right\}_{n \in \mathbb{N}} \subseteq W^{1, p} \text { is bounded. }
\end{aligned}
$$

Exploiting the fact that $W^{1, p} \hookrightarrow C\left(T, \mathbb{R}^{N}\right)$ compactly, we infer that

$$
\left\{\widehat{u}_{n}\right\}_{n \in \mathbb{N}} \subseteq C\left(T, \mathbb{R}^{N}\right) \text { is relatively compact. }
$$

We have

$$
\begin{array}{lr} 
& -a\left(\widehat{u}_{n}^{\prime}(t)\right)^{\prime}+\varphi_{p}\left(\widehat{u}_{n}\right)(t)+A_{\lambda}\left(\widehat{u}_{n}(t)\right)=h_{n}(t) \quad \text { for a.a. } t \in T, \widehat{u} \in B C, \\
& \text { for all } n \in \mathbb{N}, \\
\Rightarrow & \left\{a\left(\widehat{u}_{n}^{\prime}(t)\right)^{\prime}\right\}_{n \in \mathbb{N}} \subseteq L^{p^{\prime}} \text { is bounded (see (4)). }
\end{array}
$$

From (5) we have

$$
\begin{array}{r}
a\left(\widehat{u}_{n}^{\prime}(t)\right)=a\left(\widehat{u}_{n}^{\prime}(0)\right)+\int_{0}^{t}\left[\varphi_{p}\left(\widehat{u}_{n}\right)(s)+A_{\lambda}\left(\widehat{u}_{n}(s)\right)-h_{n}(s)\right] d s \\
\text { for all } t \in T, \text { all } n \in \mathbb{N} .
\end{array}
$$

We let $k\left(\widehat{u}_{n}\right) \in L^{p^{\prime}}$ be defined by

$$
k\left(\widehat{u}_{n}\right)(t)=h_{n}(t)-A_{\lambda}\left(\widehat{u}_{n}(t)\right)-\varphi_{p}\left(\widehat{u}_{n}\right)(t), \quad n \in \mathbb{N} .
$$

Then we have

$$
\widehat{u}_{n}^{\prime}(t)=a^{-1}\left[a\left(\widehat{u}_{n}^{\prime}(0)\right)-H\left(k\left(\widehat{u}_{n}\right)\right)(t)\right]
$$


with $H\left(k\left(\widehat{u}_{n}\right)\right)(t)=\int_{0}^{t} k\left(\widehat{u}_{n}\right)(s) d s$.

If the boundary condition $B C$ is Dirichlet or periodic, then

$$
\int_{0}^{b} \widehat{u}_{n}^{\prime}(t) d t=0 .
$$

So, we have

$$
\int_{0}^{b} a^{-1}\left[a\left(\widehat{u}_{n}^{\prime}(0)\right)-H\left(k\left(\widehat{u}_{n}\right)\right)(t)\right] d t=0 .
$$

Then by Proposition 2.2 of Manásevich-Mawhin [16], we have

$$
a\left(\widehat{u}_{n}^{\prime}(0)\right)=\widehat{\sigma}\left(H\left(k\left(\widehat{u}_{n}\right)\right)\right) \quad \text { for all } n \in \mathbb{N},
$$

with $\widehat{\sigma}: C\left(T, \mathbb{R}^{N}\right) \rightarrow \mathbb{R}^{N}$ a continuous map which sends bounded sets in $C\left(T, \mathbb{R}^{N}\right)$ to bounded sets in $\mathbb{R}^{N}$. From (4) and (7) we see that

$$
\begin{aligned}
& \left\|H\left(k\left(\widehat{u}_{n}\right)\right)\right\|_{C\left(T, \mathbb{R}^{N}\right)} \leq c_{2} \text { for some } c_{2}>0, \text { all } n \in \mathbb{N}, \\
\Rightarrow \quad & \left\{a\left(\widehat{u}_{n}^{\prime}(0)\right\}_{n \in \mathbb{N}} \subseteq \mathbb{R}^{N}\right. \text { is bounded (see (9)). }
\end{aligned}
$$

Recall that $a^{-1}$, seen as a map from $C\left(T, \mathbb{R}^{N}\right)$ into $C\left(T, \mathbb{R}^{N}\right)$, maps bounded sets to bounded sets. Therefore from (8) we see that

$$
\begin{aligned}
& \left|\widehat{u}_{n}^{\prime}(t)\right| \leq c_{3} \text { for some } c_{3}>0 \text {, all } t \in T \text {, all } n \in \mathbb{N}, \\
\Rightarrow & \left.\left|a\left(\widehat{u}_{n}^{\prime}(t)\right)\right| \leq c_{4} \text { for some } c_{4}>0, \text { all } t \in T \text {, all } n \in \mathbb{N} \text { (see hypothesis } H_{0}(\mathrm{i})\right) .
\end{aligned}
$$

Therefore from (6) and (10) it follows

$$
\left\{a\left(\widehat{u}_{n}^{\prime}(\cdot)\right)\right\}_{n \in \mathbb{N}} \subseteq W^{1, p^{\prime}} \text { is bounded. }
$$

Now suppose that the boundary condition $B C$ is Neumann or mixed. Then

$$
\begin{aligned}
& a\left(\widehat{u}_{n}^{\prime}(0)\right)=0 \quad \text { for all } n \in \mathbb{N}, \\
\Rightarrow & \widehat{u}_{n}^{\prime}(t)=a^{-1}\left(-H\left(k\left(\widehat{u}_{n}^{\prime}\right)\right)(t)\right) \quad \text { for all } t \in T, \text { all } n \in \mathbb{N}(\text { see }(8)), \\
\Rightarrow & \left|\widehat{u}_{n}^{\prime}(t)\right| \leq c_{5} \quad \text { for some } c_{5}>0, \text { all } t \in T, \text { all } n \in \mathbb{N}, \\
\Rightarrow & \left|a\left(\widehat{u}_{n}^{\prime}(t)\right)\right| \leq c_{6} \quad \text { for some } c_{6}>0, \text { all } t \in T, \text { all } n \in \mathbb{N} .
\end{aligned}
$$

Then again we infer that (11) holds.

Using (11) and recalling that $W^{1, p} \hookrightarrow C\left(T, \mathbb{R}^{N}\right)$ compactly, we infer that

$$
\begin{aligned}
& \left\{a\left(\widehat{u}_{n}^{\prime}(\cdot)\right)\right\}_{n \in \mathbb{N}} \subseteq C\left(T, \mathbb{R}^{N}\right) \text { is relatively compact, } \\
\Rightarrow & \left\{\widehat{u}_{n}^{\prime}\right\}_{n \in \mathbb{N}} \subseteq C\left(T, \mathbb{R}^{N}\right) \text { is relatively compact. }
\end{aligned}
$$

Then (4) and (12) imply that

$$
\left\{\widehat{u}_{n}\right\}_{n \in \mathbb{N}} \subseteq C^{1} \text { is relatively compact. }
$$


So, by passing to a subsequence if necessary, we may assume that

$$
\widehat{u}_{n} \rightarrow \widetilde{u} \text { in } C^{1} \text {. }
$$

Note that

$$
\left\{\begin{array}{l}
\varphi_{p}\left(\widehat{u}_{n}\right) \rightarrow \varphi_{p}(\widetilde{u}), \widehat{A}_{\lambda}\left(\widehat{u}_{n}\right) \rightarrow \widehat{A}_{\lambda}(\widetilde{u}), h_{n} \stackrel{w}{\rightarrow} h \text { in } L^{p^{\prime}}, \\
\left(-\widehat{u}_{n}, h_{n}-\varphi_{p}\left(\widehat{u}_{n}\right)-\widehat{A}_{\lambda}\left(\widehat{u}_{n}\right)\right) \in \mathrm{Gr} \widehat{a} \quad \text { for all } n \in \mathbb{N} .
\end{array}\right.
$$

Recall that $\widehat{a}(\cdot)$ is maximal monotone (see Proposition 2). Therefore

$$
\mathrm{Gr} \widehat{a} \subseteq L^{p} \times L_{w}^{p^{\prime}} \text { is closed, }
$$

with $L_{w}^{p^{\prime}}$ being the Lebesgue space $L^{p^{\prime}}\left(T, \mathbb{R}^{N}\right)$ equipped with the weak topology (see Gasiński-Papageorgiou [7], Proposition 3.2.15, p. 308). Then on account of (13), we have

$$
\begin{aligned}
& \left(-\tilde{u}, h-\varphi_{p}(\widetilde{u})-\widehat{A}_{\lambda}(\tilde{u})\right) \in \operatorname{Gr} \widehat{a}, \\
\Rightarrow & \tilde{u}=\xi_{\lambda}(h)=\widehat{u} .
\end{aligned}
$$

So, for the original sequence we have

$$
\begin{aligned}
& \widehat{u}_{n} \rightarrow \widehat{u} \text { in } C^{1}, \\
\Rightarrow & \xi_{\lambda}(\cdot) \text { is completely continuous. }
\end{aligned}
$$

Next we introduce the multivalued map $\widehat{N}: X \rightarrow 2^{L^{p^{\prime}}}$ defined by

$$
\widehat{N}(u)=N_{F}(u)-\varphi_{p}(u) \quad \text { for all } u \in X,
$$

with $N_{F}(\cdot)$ being the multivalued Nemyckii map

$$
N_{F}(u)=S_{F\left(\cdot, u(\cdot), u^{\prime}(\cdot)\right)}^{p^{\prime}}
$$

Proposition 6 If hypotheses $H_{2}$ hold, then the multifunction $\widehat{N}(\cdot)$ has values in $P_{w k c}\left(L^{p^{\prime}}\right)$ and it is usc from $X$ with the norm topology into $L^{p^{\prime}}$ with the weak topology (denoted by $\left.L_{w}^{p^{\prime}}\right)$.

Proof Note that hypotheses $\mathrm{H}_{2}$ (i), (ii), do not imply graph measurability of $F$ (see HuPapageorgiou [11], p. 226). So, it is not immediately clear that $\widehat{N}(\cdot)$ has nonempty values. To show this, we argue as follows. Let $u \in X$. Then we can find two sequences $\left\{s_{n}\right\}_{n \in \mathbb{N}}$, $\left\{r_{n}\right\}_{n \in \mathbb{N}}$ of simple functions such that

$$
\begin{cases}s_{n}(t) \rightarrow u(t), & \left|s_{n}(t)\right| \leq|u(t)| \quad \text { for a.a. } t \in T, \text { all } n \in \mathbb{N}, \\ r_{n}(t) \rightarrow u^{\prime}(t), & \left|r_{n}(t)\right| \leq\left|u^{\prime}(t)\right| \quad \text { for a.a. } t \in T, \text { all } n \in \mathbb{N} .\end{cases}
$$

We consider the multifunction $G_{n}: T \rightarrow P_{k c}\left(\mathbb{R}^{N}\right)$ defined by

$$
G_{n}(t)=F\left(t, s_{n}(t), r_{n}(t)\right) \quad \text { for all } t \in T, \text { all } n \in \mathbb{N} .
$$


Hypothesis $H_{2}(\mathrm{i})$ implies that

$$
\operatorname{Gr} G_{n} \in \mathcal{L}_{T} \otimes B\left(\mathbb{R}^{N}\right) \otimes B\left(\mathbb{R}^{N}\right) \quad \text { for all } n \in \mathbb{N}
$$

(recall that $\mathcal{L}_{T}$ is the Lebesgue $\sigma$-algebra of $T$ and $B\left(\mathbb{R}^{N}\right)$ the Borel $\sigma$-algebra of $\mathbb{R}^{N}$ ).

Invoking the Yankov-von Neumann-Aumann selection theorem (see Hu-Papageorgiou [11], Theorem 2.14, p. 158), we can find $g_{n}: T \rightarrow \mathbb{R}^{N}, n \in \mathbb{N}$, a Lebesgue measurable map such that

$$
g_{n}(t) \in G_{n}(t)=F\left(t, s_{n}(t), r_{n}(t)\right) \quad \text { for a.a. } t \in T \text {, all } n \in \mathbb{N} \text {. }
$$

Since $u \in X$, from hypothesis $H_{2}$ (iv), we have that

$$
\left\{g_{n}\right\}_{n \in \mathbb{N}} \subseteq L^{2} \text { is bounded. }
$$

So, we may assume that

$$
g_{n} \stackrel{w}{\rightarrow} g \text { in } L^{2} \text { as } n \rightarrow+\infty .
$$

On account of Proposition 3.9, p. 694, of Hu-Papageorgiou [11], we have

$$
\begin{aligned}
& g(t) \in \overline{\mathrm{conv}} \limsup _{n \rightarrow+\infty} F\left(t, s_{n}(t), r_{n}(t)\right) \quad(\text { see }(15),(16)) \\
& \subseteq F\left(t, u(t), u^{\prime}(t)\right) \quad\left(\text { see }(14) \text { and hypothesis } H_{2}(\mathrm{ii})\right) .
\end{aligned}
$$

Therefore we have that

$$
\begin{aligned}
& g \in N_{F}(u), \\
\Rightarrow \quad & \widehat{N}(u) \neq 0 \quad \text { for all } u \in X .
\end{aligned}
$$

Moreover, from hypothesis $H_{2}$ (iv) and the definition of $\widehat{N}(\cdot)$, it is clear that $\widehat{N}(\cdot)$ is $P_{w k c}\left(L^{p^{\prime}}\right)$-valued. Hypothesis $H_{2}$ (iv) shows that $\widehat{N}(\cdot)$ is locally compact into $L_{w}^{p^{\prime}}$ (see Sect. 2) and so in order to prove the desired upper semicontinuity of $\widehat{N}(\cdot)$, it suffices to show that $\widehat{N}(\cdot)$ is closed (that is, Gr $\widehat{N} \subseteq X \times L_{w}^{p^{\prime}}$ is closed). To this end, let $\left\{u_{n}\right\}_{n \in \mathbb{N}} \subseteq X$ and $\left\{\widehat{f}_{n}\right\}_{n \in \mathbb{N}} \subseteq L^{p}$ be two sequences which satisfy

$$
u_{n} \rightarrow u \text { in } X, \quad \widehat{f_{n}} \stackrel{w}{\rightarrow} f \text { in } L^{p^{\prime}}, \quad\left(u_{n}, \widehat{f_{n}}\right) \in \mathrm{Gr} \widehat{N} \text { for all } n \in \mathbb{N} .
$$

We have $\widehat{f_{n}}=f_{n}-\varphi_{p}\left(u_{n}\right)$ with $f_{n} \in N_{F}\left(u_{n}\right)$ for all $n \in \mathbb{N}$. We have

$$
\begin{aligned}
& \varphi_{p}\left(u_{n}\right) \rightarrow \varphi_{p}(u) \text { in } L^{p^{\prime}} \\
& \Rightarrow \quad f_{n} \stackrel{w}{\rightarrow} f=\widehat{f}+\varphi_{p}(u) \text { in } L^{p^{\prime}}(\text { see }(17)) .
\end{aligned}
$$

Then as before, using Proposition 3.9, p. 694, of Hu-Papageorgiou [11], we have

$$
\begin{aligned}
& \quad f(t) \in \overline{\operatorname{conv}} \limsup _{n \rightarrow+\infty} F\left(t, u_{n}(t), u_{n}^{\prime}(t)\right) \subseteq F\left(t, u(t), u^{\prime}(t)\right) \\
& \quad\left(\text { see (17) and hypothesis } H_{2}(\mathrm{ii})\right), \\
& \Rightarrow \quad f \in N_{F}(u),
\end{aligned}
$$




$$
\begin{aligned}
& \Rightarrow \widehat{f}=f-\varphi_{p}(u) \in \widehat{N}(u) \\
& \Rightarrow \widehat{N}(\cdot) \text { is closed and so usc as claimed by the Proposition. }
\end{aligned}
$$

Consider the map $L_{\lambda}: X \rightarrow 2^{X} \backslash\{\emptyset\}$ defined by

$$
L_{\lambda}(u)=\left[\xi_{\lambda} \circ \widehat{N}\right](u) \quad \text { for all } u \in X .
$$

Evidently a fixed point of $L_{\lambda}(\cdot)$ is a solution of problem $\left(2_{\lambda}\right)$. To produce such a fixed point, we will use Theorem 1.

Proposition 7 If hypotheses $H_{0}, H_{1}, H_{2}$ hold and $\lambda>0$, then problem $\left(2_{\lambda}\right)$ admits a solution $\tilde{u}_{\lambda} \in X$.

Proof We consider the set

$$
K=\left\{u \in X: u \in \mu\left(\xi_{\lambda} \circ \widehat{N}\right)(u), 0<\mu<1\right\} .
$$

For $u \in K$, we have

$$
\frac{1}{\mu} u=\xi_{\lambda}(\widehat{f}) \quad \text { with } \widehat{f} \in \widehat{N}(u) .
$$

Then $\widehat{f}=f-\varphi_{p}(u)$ with $f \in N_{F}(u)$. It follows that

$$
\widehat{a}\left(\frac{1}{\mu} u^{\prime}\right)+\varphi_{p}\left(\frac{1}{\mu} u\right)+\widehat{A}_{\lambda}\left(\frac{1}{\mu} u\right)=-\widehat{f} .
$$

Acting with $u \in X$, performing integration by parts and using hypothesis $H_{0}$ (ii) and the fact that $\left(A_{\lambda}(x), x\right)_{\mathbb{R}^{N}} \geq 0$ for all $x \in \mathbb{R}^{N}$, we obtain

$$
\frac{c_{0}}{\mu}\left\|u^{\prime}\right\|_{p}^{p}+\left[\frac{1}{\mu^{p}}-1\right]\|u\|_{p}^{p} \leq \int_{0}^{b}(-f, u)_{\mathbb{R}^{N}} d t \quad(\text { see }(18)) .
$$

On account of hypotheses $H_{2}$ (iii), (iv), given $\varepsilon>0$, we can find $k_{\varepsilon} \in L^{2} \subseteq L^{p^{\prime}}$ (recall $p \geq 2$ ) such that

$$
(-f(t), u(t))_{\mathbb{R}^{N}} \leq[-\vartheta(t)+\varepsilon]|u(t)|^{p}+k_{\varepsilon}(t) \quad \text { for a.a. } t \in T .
$$

We return to (19) and use (20). Then

$$
c_{0}\left\|u^{\prime}\right\|_{p}^{p}+\int_{0}^{b} \vartheta(t)|u|^{p} d t-\varepsilon\|u\|^{p} \leq c_{7} \quad \text { for some } c_{7}>0(\text { recall } 0<\mu<1),
$$

$\Rightarrow \quad\left[c_{8}-\varepsilon\right]\|u\|^{p} \leq c_{7} \quad$ for some $c_{8}>0$ (see Mugnai-Papageorgiou [18], Lemma 4.11).

Choosing $\varepsilon \in\left(0, c_{8}\right)$, we conclude that

$$
K \subseteq W^{1, p} \text { is bounded. }
$$

Then as in the proof of Proposition 5, we obtain that

$K \subseteq X$ is bounded (in fact relatively compact). 
So, we can apply Theorem 1 and find $\widetilde{u}_{\lambda} \in W^{1, p}$ such that

$$
\begin{aligned}
& \tilde{u}_{\lambda} \in L_{\lambda}\left(\tilde{u}_{\lambda}\right), \\
\Rightarrow \quad & \tilde{u}_{\lambda} \in X \text { is a solution of problem }\left(2_{\lambda}\right) .
\end{aligned}
$$

Finally we will let $\lambda \rightarrow 0^{+}$to produce a solution for problem (1). To perform this passage to the limit as $\lambda \rightarrow 0^{+}$, we will need the next lemma, which can be found in a more general form in Gasiński-Papageorgiou [9], Lemma 2.3. Consider the "lifting" of $A(\cdot)$ on $\left(L^{p}, L^{p^{\prime}}\right)$, that is, the operator $\widehat{A}: L^{p} \rightarrow 2^{L^{p^{\prime}}}$ defined by

$$
\widehat{A}(u)=\left\{u^{*} \in L^{p^{\prime}}: u^{*}(t) \in A(u(t)) \text { for a.a. } t \in T\right\},
$$

with $D(\widehat{A})=\left\{u \in L^{p}: S_{A(u(\cdot))}^{p^{\prime}} \neq \emptyset\right\}$.

Lemma 1 The "lifting" operator $\widehat{A}: L^{p} \rightarrow 2^{L^{p^{\prime}}}$ is maximal monotone.

Now we are ready for the existence result for the convex problem.

Theorem 3 If hypotheses $H_{0}, H_{1}, H_{2}$ hold, then problem (1) admits a solution $\tilde{u} \in X$.

Proof Let $\lambda_{n} \rightarrow 0^{+}$and let $\tilde{u}_{n}=\tilde{u}_{\lambda_{n}} \in X$ be a solution of problem $\left(2_{\lambda_{n}}\right), n \in \mathbb{N}$ (see Proposition 7). We have

$$
\widehat{a}\left(\widetilde{u}_{n}\right)+\widehat{A}_{\lambda_{n}}\left(\widetilde{u}_{n}\right)+f_{n}=0 \text { with } f_{n} \in N_{F}\left(\widetilde{u}_{n}\right) \text { for all } n \in \mathbb{N} .
$$

Acting with $\tilde{u}_{n} \in X$, performing integration by parts and using hypothesis $H_{0}$ (ii) and the fact that $\left(A_{\lambda_{n}}(x), x\right)_{\mathbb{R}^{N}} \geq 0$ for all $x \in \mathbb{R}^{N}$, all $n \in \mathbb{N}$ (see hypotheses $H_{1}$ ), we obtain

$$
\begin{aligned}
& c_{0}\left\|\widetilde{u}_{n}^{\prime}\right\|_{p}^{p} \leq \int_{0}^{b}\left(-f_{n}, \widetilde{u}_{n}\right)_{\mathbb{R}^{N}} d t, \\
\Rightarrow & c_{0}\left\|\widetilde{u}_{n}^{\prime}\right\|_{p}^{p}+\int_{0}^{b} \vartheta(t)\left|\widetilde{u}_{n}\right|^{p} d t-\varepsilon\left\|\widetilde{u}_{n}\right\|^{p} \leq c_{9} \quad \text { for some } c_{9}>0 \text { (see (20)), } \\
\Rightarrow & \left\{\widetilde{u}_{n}\right\}_{n \in \mathbb{N}} \subseteq W^{1, p} \text { is bounded (choosing } \varepsilon>0 \text { small, see the proof of Proposition 7). }
\end{aligned}
$$

So, we may assume that

$$
\begin{aligned}
\tilde{u}_{n} \stackrel{w}{\rightarrow} \tilde{u} \text { in } W^{1, p} \text { and } \tilde{u}_{n} \rightarrow \tilde{u} \text { in } C\left(T, \mathbb{R}^{N}\right) \\
\text { (recall that } W^{1, p} \hookrightarrow C\left(T, \mathbb{R}^{N}\right) \text { compactly). }
\end{aligned}
$$

On account of Proposition $1, t \rightarrow A_{\lambda_{n}}\left(\tilde{u}_{n}(t)\right)$ is Lipschitz continuous on $T$ and so by Rademacher's theorem (see Gasiński-Papageorgiou [7], p. 56), it is differentiable almost everywhere. On (21) we act with $A_{\lambda_{n}}\left(\widetilde{u}_{n}\right)$ and have

$$
\int_{0}^{b}\left(-a\left(\widetilde{u}_{n}^{\prime}\right)^{\prime}, A_{\lambda_{n}}\left(\widetilde{u}_{n}\right)\right)_{\mathbb{R}^{N}} d t+\left\|\widehat{A}_{\lambda_{n}}\left(\widetilde{u}_{n}\right)\right\|_{2}^{2} \leq \int_{0}^{b}\left|f_{n} \| A_{\lambda_{n}}\left(u_{n}\right)\right| d t \quad \text { for all } n \in \mathbb{N} .
$$

Performing integration by parts and using the fact that $A_{\lambda_{n}}(0)=0$, we obtain

$$
\int_{0}^{b}\left(-a\left(\tilde{u}_{n}^{\prime}\right)^{\prime}, A_{\lambda_{n}}\left(\tilde{u}_{n}\right)\right)_{\mathbb{R}^{N}} d t=\int_{0}^{b}\left(a\left(\tilde{u}_{n}^{\prime}\right), \frac{d}{d t} A_{\lambda_{n}}\left(\widetilde{u}_{n}\right)\right)_{\mathbb{R}^{N}} d t .
$$


By the chain rule (see Leoni [14], Corollary 3.52, p. 97), we have

$$
\frac{d}{d t} A_{\lambda_{n}}\left(\widetilde{u}_{n}(t)\right)=A_{\lambda_{n}}^{\prime}\left(\widetilde{u}_{n}(t)\right) \tilde{u}_{n}^{\prime}(t) \quad \text { for a.a. } t \in T
$$

Therefore we obtain

$$
\int_{0}^{b}\left(a\left(\widetilde{u}_{n}^{\prime}\right), \frac{d}{d t} A_{\lambda_{n}}\left(\widetilde{u}_{n}\right)\right)_{\mathbb{R}^{N}} d t=\int_{0}^{b} a_{0}\left(\left|\widetilde{u}_{n}^{\prime}\right|\right)\left(\widetilde{u}_{n}^{\prime}, A_{\lambda_{n}}^{\prime}\left(\widetilde{u}_{n}\right) \widetilde{u}_{n}^{\prime}\right)_{\mathbb{R}^{N}} d t .
$$

The monotonicity of $A_{\lambda_{n}}(\cdot)$ (see Proposition 1) implies that

$$
\begin{aligned}
& \left(x, A_{\lambda_{n}}^{\prime}\left(u_{n}\right) x\right)_{\mathbb{R}^{N}} \geq 0 \quad \text { for all } x \in \mathbb{R}^{N}, \text { all } n \in \mathbb{N}, \\
\Rightarrow & \int_{0}^{b}\left(-a\left(\widetilde{u}_{n}^{\prime}\right)^{\prime}, A_{\lambda_{n}}\left(\widetilde{u}_{n}\right)\right)_{\mathbb{R}^{N}} d t \geq 0 \quad \text { (see (24), (25)). }
\end{aligned}
$$

Using (26) in (23), we have

$$
\left\|\widehat{A}_{\lambda_{n}}(\widetilde{u})\right\|_{2}^{2} \leq\left\|f_{n}\right\|_{2}\left\|\widehat{A}_{\lambda_{n}}\left(\tilde{u}_{n}\right)\right\|_{2} \quad \text { for all } n \in \mathbb{N} .
$$

But from (22) and hypothesis $H_{2}($ iv), we see that

$$
\begin{aligned}
& \left\{f_{n}\right\}_{n \in \mathbb{N}} \subseteq L^{2} \text { is bounded, } \\
\Rightarrow \quad & \left.\left\{\widehat{A}_{\lambda_{n}}\left(\widetilde{u}_{n}\right)\right\}_{n \in \mathbb{N}} \subseteq L^{2} \text { is bounded (see }(27)\right) .
\end{aligned}
$$

So, we may assume that

$$
\begin{aligned}
& \widehat{A}_{\lambda_{n}}\left(\widetilde{u}_{n}\right) \stackrel{w}{\rightarrow} \eta \text { in } L^{2} \text { as } n \rightarrow+\infty \\
& \text { and } \quad f_{n} \stackrel{w}{\rightarrow} f \text { in } L^{2} \text { as } n \rightarrow+\infty .
\end{aligned}
$$

Then as before (see the proof of Proposition 6), using (29) and hypothesis $H_{2}$ (ii), we show that $f \in N_{F}(\tilde{u})$.

From (21) we have

$$
\left(\widetilde{u}_{n},-\widehat{A}_{\lambda_{n}}\left(\widetilde{u}_{n}\right)-f_{n}\right) \in \operatorname{Gr} \widehat{a} \text { for all } n \in \mathbb{N} .
$$

From Proposition 2 we know that $\widehat{a}(\cdot)$ is maximal monotone. Hence from (22), (28), (29) and (30), it follows that

$$
\begin{aligned}
& (\tilde{u},-\eta-f) \in \operatorname{Gr} \widehat{a}, \\
\Rightarrow & \widehat{a}(\tilde{u})+\eta-f=0 \quad \text { with } f \in N_{F}(\widetilde{u}) .
\end{aligned}
$$

To finish the proof, we need to show that $\eta \in \widehat{A}(\widetilde{u})$.

Let $\widehat{J}_{\lambda_{n}}(u)(\cdot)=J_{\lambda_{n}}(u(\cdot))$ for all $u \in L^{p}$ (see Sect. 2). We have

$$
\begin{aligned}
& \widehat{J}_{\lambda_{n}}\left(\widetilde{u}_{n}\right)+\lambda_{n} \widehat{A}_{\lambda_{n}}\left(\widetilde{u}_{n}\right)=\widetilde{u}_{n} \quad \text { for all } n \in \mathbb{N}, \\
\Rightarrow & \widehat{J}_{\lambda_{n}}\left(\tilde{u}_{n}\right) \rightarrow \tilde{u} \text { in } L^{2},
\end{aligned}
$$

(note that $\left\{\widehat{A}_{\lambda_{n}}\left(\widetilde{u}_{n}\right)\right\}_{n \in \mathbb{N}} \subseteq L^{2}$ is bounded and $\lambda_{n} \rightarrow 0^{+}$). 
We know that

$$
\left(\widehat{J}_{\lambda_{n}}\left(\widetilde{u}_{n}\right), \widehat{A}_{\lambda_{n}}\left(\widetilde{u}_{n}\right)\right) \in \operatorname{Gr} \widehat{A} \text { for all } n \in \mathbb{N} \text { (see Proposition 1). }
$$

Since $\widehat{A}(\cdot)$ is maximal monotone (see Lemma 1), from (28) and (31) we infer that

$$
\begin{aligned}
& (\tilde{u}, \eta) \in \operatorname{Gr} \widehat{A}, \\
\Rightarrow \quad & \eta \in \widehat{A}(\widetilde{u}),
\end{aligned}
$$

which is what we wanted. So, we conclude that $\tilde{u} \in X$ is a solution of problem (1).

\section{Nonconvex Problem}

In this section, we prove an existence theorem for the "nonconvex problem", that is, the multivalued perturbation $F(t, x, y)$ has nonconvex values (hypotheses $H_{3}$ ).

Theorem 4 If hypotheses $H_{0}, H_{1}, H_{3}$ hold, then problem (1) admits a solution $\tilde{u} \in X$.

Proof We consider the multifunction $N_{F}: X \rightarrow 2^{L^{p^{\prime}}}$ (recall that $2 \leq p$ ) defined by

$$
N_{F}(u)=S_{F\left(\cdot, u(\cdot), u^{\prime}(\cdot)\right)}^{p^{\prime}} \quad \text { for all } u \in X
$$

Hypotheses $H_{3}(\mathrm{i})$, (iv) imply that $N_{F}(\cdot)$ has values in $P_{f}\left(L^{p^{\prime}}\right)$.

Claim: $N_{F}(\cdot)$ is lsc.

From Sect. 2 (see also Proposition 2.26, p. 45, of Hu-Papageorgiou [11]), we know that in order to prove the Claim, it suffices to show that for every $g \in L^{p^{\prime}}$, the function

$$
u \rightarrow d\left(g, N_{F}(u)\right)=\inf \left[\|g-f\|_{p^{\prime}}: f \in N_{F}(u)\right]
$$

is upper semicontinuous (as an $\mathbb{R}_{+}$-valued function). We have

$$
\begin{aligned}
d\left(g, N_{F}(u)\right)^{p^{\prime}} & =\inf \left[\int_{0}^{b}|g-f|^{p^{\prime}} d t: f \in N_{F}(u)\right] \\
& =\int_{0}^{b} d\left(g(t), F\left(t, u(t), u^{\prime}(t)\right)\right)^{p^{\prime}} d t
\end{aligned}
$$

(see Theorem 3.24, p. 183, of Hu-Papageorgiou [11]).

Let $\widehat{d}_{g}(u)=d\left(g, N_{F}(u)\right)^{p^{\prime}}$. We need to show that for all $\mu \geq 0$, the set

$$
U_{\mu}=\left\{u \in X: \widehat{d}_{g}(u) \geq \mu\right\} \text { is closed. }
$$

So, let $\left\{u_{n}\right\}_{n \in \mathbb{N}} \subseteq U_{\mu}$ and assume that $u_{n} \rightarrow u$ in $X$. We have

$$
\widehat{d}_{g}\left(u_{n}\right) \geq \mu \quad \text { for all } n \in \mathbb{N} .
$$

We have

$$
u_{n} \rightarrow u \text { and } u_{n}^{\prime} \rightarrow u^{\prime} \text { in } C\left(T, \mathbb{R}^{N}\right)
$$


Then from (32) and passing to the limit as $n \rightarrow+\infty$, on account of (33) and Fatou's lemma, we have

$$
\begin{aligned}
\mu \leq \limsup _{n \rightarrow+\infty} \widehat{d}_{g}\left(u_{n}\right) & \leq \int_{0}^{b} \limsup _{n \rightarrow+\infty} d\left(g(t), F\left(t, u_{n}(t), u_{n}^{\prime}(t)\right)\right)^{p^{\prime}} d t \\
& \leq \int_{0}^{b} d\left(g(t), F\left(t, u(t), u^{\prime}(t)\right)\right)^{p^{\prime}} d t \\
& \text { (see (33) and hypothesis } \left.H_{3}(\mathrm{ii})\right) \\
& =\widehat{d}_{g}(u) \\
\Rightarrow \quad & u \in U_{\mu} \text { and so } U_{\mu} \text { is closed. }
\end{aligned}
$$

This proves the Claim.

Since $N_{F}(\cdot)$ has decomposable values in $P_{f}\left(L^{p^{\prime}}\right)$ and it is lsc (see the Claim), we can use Theorem 2 and produce a continuous map $e: X \rightarrow L^{p^{\prime}}$ such that

$$
e(u) \in N_{F}(u) \text { for all } u \in X .
$$

Then we consider the following boundary value problem

$$
\left\{\begin{array}{l}
a\left(u^{\prime}(t)\right)^{\prime} \in A(u(t))+e(u)(t) \quad \text { for a.a. } t \in T, \\
u \in B C .
\end{array}\right.
$$

Reasoning as in the "convex" case (see Sect. 3). We show that problem (34) admits a solution $\tilde{u} \in X$. Evidently $\tilde{u} \in X$ is also a solution of (1).

\section{Relaxation}

We introduce the following two sets:

$$
\begin{aligned}
& S_{c}=\text { solution set of the "convex" problem, } \\
& S=\text { solution set of the "nonconvex" problem. }
\end{aligned}
$$

Our aim in this section, is to determine conditions which guarantee that $\bar{S}^{\|\cdot\|}=S_{c}$. Such a result is known in the literature as "relaxation theorem" and has important consequences in many applied areas such as control theory and game theory.

Our solution to this fundamental problem is partial. We prove a relaxation theorem only for the Dirichlet problem, under stronger conditions on the map $a(\cdot)$. When we try to extend the result to other boundary conditions, we encounter serious technical difficulties and it is an interesting open problem whether it is possible to have a relaxation theorem for the Neumann, periodic and mixed problems. Also, we will consider multivalued perturbations $F$ which are independent of the derivative $u^{\prime}$. So, now the problem under consideration is the following

$$
\left\{\begin{array}{l}
a\left(u^{\prime}(t)\right)^{\prime} \in A(u(t))+F(t, u(t)) \quad \text { for a.a. } t \in T, \\
u(0)=u(b)=0 .
\end{array}\right.
$$

The condition on the data of problem (35) are the following: 
$H_{4}: a: \mathbb{R}^{N} \rightarrow \mathbb{R}^{N}$ has the form $a(y)=a_{0}(|y|) y$ for all $y \in \mathbb{R}^{N}$, with $a_{0}(t)>0$ for all $t>0$ and

(i) $a(\cdot)$ is continuous and there exists $\widehat{c}>0$ such that

$$
\widehat{c}\left|y-y^{\prime}\right|^{2} \leq\left(a(y)-a\left(y^{\prime}\right), y-y^{\prime}\right)_{\mathbb{R}^{N}} \text { for a.a. } t \in T \text {, all } y, y^{\prime} \in \mathbb{R}^{N} ;
$$

(ii) there exists $c_{0}>0$ such that

$$
c_{0}|y|^{p} \leq(a(y), y)_{\mathbb{R}^{N}} \quad \text { for all } y \in \mathbb{R}^{N}, 2 \leq p .
$$

Example 2 The following maps satisfy hypotheses $H_{4}$ :

(a) $a(y)=|y|^{p-2} y+y, 2<p<+\infty$.

(b) $a(y)=y+\left[1+|y|^{2}\right]^{\frac{p-2}{2}}, 2<p<+\infty$.

(c) $a(y)=y+|y|^{p-2} y\left(\ln \left(1+|y|^{p}\right)+\frac{|y|^{p}}{1+|y|^{p}}\right), 2<p<+\infty$.

The hypotheses on the multivalued perturbation $F(t, x)$ are the following:

$H_{5}: F: T \times \mathbb{R}^{N} \rightarrow P_{k}\left(\mathbb{R}^{N}\right)$ is a multifunction such that

(i) for every $x \in \mathbb{R}^{N}, t \rightarrow F(t, x)$ is graph measurable;

(ii) $h(F(t, x), F(t, v)) \leq k(t)|x-v|$ for a.a. $t \in T$, all $x, v \in \mathbb{R}^{N}$, with $k \in L^{1}(T)$ such that $b\|k\|_{1}<\widehat{c}$;

(iii) if $\sigma_{0}(t, x)=\inf \left[(v, x)_{\mathbb{R}^{N}}: v \in F(t, x)\right]$, then

$$
\liminf _{|x| \rightarrow+\infty} \frac{\sigma_{0}(t, x)}{|x|^{p}} \geq \vartheta(t) \quad \text { uniformly for a.a. } t \in T,
$$

with $\vartheta \in L^{\infty}(T), \vartheta(t) \geq-c_{0} \widehat{\lambda}_{1}$ for a.a. $t \in T, \vartheta \not \equiv-c_{0} \widehat{\lambda}_{1}$;

(iv) for every $M>0$, there exists $\gamma_{M} \in L^{2}(T)$ such that

$$
|F(t, x)| \leq \gamma_{M}(t) \quad \text { for a.a. } t \in T \text {, all }|x| \leq M .
$$

The "convex problem" is obtained by replacing $F$ with $\overline{\operatorname{conv}} F$. We start by mentioning that the set $S_{c} \subseteq C^{1}$ is closed. This follows easily from the proof of the "convex" existence theorem. This observation is valid for the more general setting of Sect. 3 .

In what follows the solution sets $S_{c}$ and $S$ refer to problem (35).

Theorem 5 If hypotheses $H_{4}, H_{1}, H_{5}$ hold, then $S_{c}=\bar{S}^{W^{1,2}}$.

Proof Let $u \in S_{c}$. Then we have

$$
\left\{\begin{array}{l}
a\left(u^{\prime}(t)\right)^{\prime} \in A(u(t))+f(t) \quad \text { for a.a. } t \in T, \\
u(0)=u(b)=0,
\end{array}\right.
$$

with $f \in S_{\overline{\operatorname{conv}} F(\cdot, u(\cdot))}^{p^{\prime}}$.

Invoking Proposition 3.30, p. 185, of Hu-Papageorgiou [11], we can find $g_{n} \in S_{F(\cdot, u(\cdot))}^{p^{\prime}}$ such that

$$
g_{n} \stackrel{w}{\rightarrow} f \text { in } L^{p^{\prime}}
$$


Let $\varepsilon_{n} \rightarrow 0^{+}, y \in W^{1, p}$ and consider the multifunction

$$
G_{n}(t)=\left\{v \in \mathbb{R}^{N}:\left|g_{n}(t)-v\right|<\varepsilon_{n}+d\left(g_{n}(t), F(t, y(t))\right), v \in F(t, y(t))\right\} .
$$

Hypotheses $H_{5}(\mathrm{i})$, (ii) imply that $F(\cdot, \cdot)$ is graph measurable (see Hu-Papageorgiou [11], Proposition 7.9, p. 229). So, it follows that

$$
\operatorname{Gr} G_{n} \in \mathcal{L}_{T} \otimes B\left(\mathbb{R}^{N}\right) .
$$

Then applying the Yankov-von Neumann-Aumann selection theorem, we can find a measurable selection of the multifunction $G_{n}(\cdot)$. Evidently this selection belongs in $L^{p^{\prime}}$ (see hypothesis $H_{5}$ (iv)).

Consider the multifunction $K_{n}: W^{1, p} \rightarrow 2^{L^{p^{\prime}}}$ defined by

$$
K_{n}(y)=\left\{h \in S_{F(\cdot, y(\cdot))}^{p^{\prime}}:\left|g_{n}(t)-h(t)\right|<\varepsilon_{n}+d\left(g_{n}(t), F(t, y(t))\right) \text {, for a.a. } t \in T\right\} .
$$

Since $L^{p^{\prime}}$ is reflexive, we see that

$$
K_{n}(y) \in P_{w k c}\left(L^{p^{\prime}}\right) \quad \text { for all } y \in W^{1, p} .
$$

Lemma 3.9, p. 239, of Hu-Papageorgiou [11], implies that $K_{n}(\cdot)$ is 1sc. Also, it has decomposable values. Therefore

$$
y \rightarrow \overline{K_{n}(y)} \|^{\|\cdot\|_{p^{\prime}}} \text { is lsc with decomposable values. }
$$

So, we can apply Theorem 2 and find a continuous map $\beta_{n}: W^{1, p} \rightarrow L^{p^{\prime}}$ such that

$$
\beta_{n}(y) \in{\overline{K_{n}(y)}}^{\|\cdot\|_{p^{\prime}}} \quad \text { for all } y \in W^{1, p} .
$$

We consider the following auxiliary Dirichlet problem

$$
\left\{\begin{array}{l}
a\left(v^{\prime}(t)\right)^{\prime} \in A(v(t))+\beta_{n}(v)(t) \quad \text { for a.a. } t \in T, \\
v(0)=v(b)=0 .
\end{array}\right.
$$

We know that problem (37) admits a solution $v_{n} \in C^{1}\left(T, \mathbb{R}^{N}\right.$ ) (see the proof of Theorem 4).

Claim: $\left\{v_{n}\right\}_{n \in \mathbb{N}} \subseteq W^{1, p}$ is bounded.

In (37) we take inner product of both sides with $-v_{n}(t)$, integrate over $T=[0, b]$, perform integration by parts and use the fact that $(A(x), x)_{\mathbb{R}^{N}} \geq 0$ for all $x \in \mathbb{R}^{N}$. Then

$$
c_{0}\left\|v_{n}^{\prime}\right\|_{p}^{p} \leq \int_{0}^{b}\left(a\left(v_{n}^{\prime}\right), v_{n}^{\prime}\right)_{\mathbb{R}^{N}} d t \leq \int_{0}^{b}\left(\beta_{n}\left(v_{n}\right),-v_{n}\right)_{\mathbb{R}^{N}} d t
$$

for all $n \in \mathbb{N}$ (see hypothesis $H_{4}(\mathrm{ii})$ ).

Hypotheses $H_{5}$ (iii), (iv) imply that given $\varepsilon>0$, we can find $\gamma_{\varepsilon} \in L^{1}(T)$ such that

$$
\left(\beta_{n}\left(v_{n}\right)(t),-v_{n}(t)\right)_{\mathbb{R}^{N}} \leq[-\vartheta(t)+\varepsilon]\left|v_{n}(t)\right|^{p}+\gamma_{\varepsilon}(t) \quad \text { for a.a. } t \in T, \text { all } n \in \mathbb{N} .
$$


Using (39) in (38), we obtain

$$
\begin{aligned}
& c_{0}\left\|v_{n}^{\prime}\right\|_{p}^{p}+\int_{0}^{b} \vartheta(t)\left|v_{n}\right|^{p} d t-\varepsilon\left\|v_{n}\right\|^{p} \leq\left\|\gamma_{\varepsilon}\right\|_{1}, \\
\Rightarrow \quad & {\left[c_{10}-\varepsilon\right]\left\|v_{n}\right\|^{p} \leq\left\|\gamma_{\varepsilon}\right\|_{1} \quad \text { for some } c_{10}>0, \text { all } n \in \mathbb{N} . }
\end{aligned}
$$

Choosing $\varepsilon \in\left(0, c_{10}\right)$, we conclude that

$$
\left\{v_{n}\right\}_{n \in \mathbb{N}} \subseteq W^{1, p} \text { is bounded. }
$$

This proves the Claim.

On account of the Claim we may assume that

$$
v_{n} \stackrel{w}{\rightarrow} v \text { in } W^{1, p} \text { and } v_{n} \rightarrow v \text { in } C\left(T, \mathbb{R}^{N}\right) .
$$

Since $A(\cdot)$ is monotone, we have

$$
\begin{aligned}
& \int_{0}^{b}\left(a\left(u^{\prime}\right)^{\prime}-a\left(v_{n}^{\prime}\right)^{\prime}, v_{n}-u\right)_{\mathbb{R}^{N}} d t \\
\leq & \int_{0}^{b}\left(h-\beta_{n}\left(v_{n}\right), v_{n}-u\right)_{\mathbb{R}^{N}} d t \\
= & \int_{0}^{b}\left(h-g_{n}, v_{n}-u\right)_{\mathbb{R}^{N}} d t+\int_{0}^{b}\left(g_{n}-\beta_{n}\left(v_{n}\right), v_{n}-u\right)_{\mathbb{R}^{N}} d t .
\end{aligned}
$$

Performing integration by parts and using hypothesis $H_{4}(\mathrm{i})$ we have

$$
\widehat{c}\left\|v_{n}^{\prime}-u^{\prime}\right\|_{2}^{2} \leq \int_{0}^{b}\left(a\left(v_{n}^{\prime}\right)-a\left(u^{\prime}\right), v_{n}^{\prime}-u^{\prime}\right)_{\mathbb{R}^{N}} d t=\int_{0}^{b}\left(a\left(u^{\prime}\right)^{\prime}-a\left(v_{n}^{\prime}\right)^{\prime}, v_{n}-u\right)_{\mathbb{R}^{N}} d t .
$$

Also we have

$$
\int_{0}^{b}\left(h-g_{n}, v_{n}-u\right)_{\mathbb{R}^{N}} d t \rightarrow 0 \quad(\text { see }(36),(40)) .
$$

Moreover, using hypothesis $H_{5}$ (ii), we obtain

$$
\begin{aligned}
& \int_{0}^{b}\left(g_{n}-\beta_{n}\left(v_{n}\right), v_{n}-u\right)_{\mathbb{R}^{N}} d t \\
\leq & \int_{0}^{b}\left[\varepsilon_{n}+h\left(F(t, u), F\left(t, v_{n}\right)\right)\right]\left|v_{n}-u\right| d t \\
\leq & \varepsilon_{n} b+\int_{0}^{b} k(t)\left|v_{n}-u\right|^{2} d t \text { for all } n \in \mathbb{N} .
\end{aligned}
$$

Note that

$$
\begin{gathered}
v_{n}(t)-u(t)=\int_{0}^{t}\left(v_{n}-u\right)^{\prime} d s, \\
\Rightarrow \quad\left|v_{n}(t)-u(t)\right| \leq \int_{0}^{t}\left|v_{n}^{\prime}-u^{\prime}\right| d s,
\end{gathered}
$$




$$
\Rightarrow \quad\left|v_{n}(t)-u(t)\right|^{2} \leq b \int_{0}^{b}\left|v_{n}^{\prime}-u^{\prime}\right|^{2} d s \quad \text { (using Jensen's inequality). }
$$

Using this last inequality in (44), we have

$$
\int_{0}^{b}\left(g_{n}-\beta_{n}\left(v_{n}\right), v_{n}-u\right)_{\mathbb{R}^{N}} d t \leq \varepsilon_{n} b+b\|k\|_{1}\left\|v_{n}^{\prime}-u^{\prime}\right\|_{2}^{2} \quad \text { for all } n \in \mathbb{N} .
$$

Using (42), (43), (45) in (41), we have

$$
\begin{aligned}
& \widehat{c}\left\|v_{n}^{\prime}-u^{\prime}\right\|_{2}^{2} \leq \varepsilon_{n}^{\prime}+b\|k\|_{1}\left\|v_{n}^{\prime}-u^{\prime}\right\|_{2}^{2} \quad \text { with } \varepsilon_{n}^{\prime} \rightarrow 0^{+}, \\
\Rightarrow & c_{11}\left\|v_{n}^{\prime}-u^{\prime}\right\|_{2}^{2} \leq \varepsilon_{n}^{\prime} \quad \text { for all } n \in \mathbb{N} \text {, some } c_{11}>0\left(\text { recall that } b\|k\|_{1}<\widehat{c}\right), \\
\Rightarrow & v_{n}^{\prime} \rightarrow u^{\prime} \text { in } L^{2}, \\
\Rightarrow & v_{n} \rightarrow u \text { in } W^{1,2} \quad(\text { see }(40) \text { and recall } p \geq 2) .
\end{aligned}
$$

Since $v_{n} \in S$ for all $n \in \mathbb{N}$ (see (37)), we conclude that

$$
S_{c}=\bar{S}^{W^{1,2}}
$$

\section{$6 \operatorname{Dom} A=\mathbb{R}^{N}$}

When $\operatorname{dom} A=\mathbb{R}^{N}$ and we restrict ourselves to the Dirichlet, Neumann and periodic problems, we can relax some of the hypotheses on the data of (1). More precisely, the new hypotheses are the following:

$H_{6}: a: \mathbb{R}^{N} \rightarrow \mathbb{R}^{N}$ is a map such that $a(0)=0$ and

(i) $a(\cdot)$ is continuous and strictly monotone;

(ii) $|a(y)| \leq c_{12}\left[1+|y|^{p-1}\right]$ for all $y \in \mathbb{R}^{N}$, some $c_{12}>0$;

(iii) $c_{0}|y|^{p} \leq(a(y), y)_{\mathbb{R}^{N}}$ for all $y \in \mathbb{R}^{N}$, some $c_{0}>0$ and $1<p<+\infty$.

Remark 6 We do not require that $a(y)=a_{0}(|y|) y$ and we remove the restriction $p \geq 2$. So, our formulation includes the singular vectorial $p$-Laplacian $|y|^{p-2} y, 1<p<2$. Another example which is covered by $H_{6}$ but not by $H_{0}$, is the map

$$
a(y)=|y|^{p-2} y+\operatorname{proj}(y, C),
$$

with $C \in P_{f c}\left(\mathbb{R}^{N}\right), 0 \in C, \operatorname{proj}(y, C)$ being the metric projection and $1<p<+\infty$.

$H_{7}: A: \mathbb{R}^{N} \rightarrow 2^{\mathbb{R}^{N}}$ is a maximal monotone map with $D(A)=\mathbb{R}^{N}, 0 \in A(0)$.

Remark 7 Even with this more restrictive condition on $A(\cdot)$, we continue to include in our framework many important classes of systems, such as gradient systems with a potential which is in general nonsmooth. In this case $A=\partial \varphi$ with $\partial \varphi$ being the convex subdifferential of a continuous convex function $\varphi(\cdot)$.

For the "convex" and "nonconvex" problems, we can improve the growth hypotheses. 
$H_{8}: F: T \times \mathbb{R}^{N} \times \mathbb{R}^{N} \rightarrow P_{k c}\left(\mathbb{R}^{N}\right)$ is a multifunction such that hypotheses $H_{8}(\mathrm{i})$, (ii), (iii) are the same as the corresponding hypotheses $H_{2}(\mathrm{i})$, (ii), (iii) and

(iv) for every $r>0$, there exist $\gamma_{r} \in L^{p^{\prime}}(T)$ and $c_{r}>0$ such that

$$
|F(t, x, y)| \leq \gamma_{r}(t)+c_{r}|y|^{p-1}
$$

for a.a. $t \in T$, all $|x| \leq r$, all $y \in \mathbb{R}^{N}$.

$H_{9}: F: T \times \mathbb{R}^{N} \times \mathbb{R}^{N} \rightarrow P_{k}\left(\mathbb{R}^{N}\right)$ is a multifunction such that hypotheses $H_{9}$ (i), (ii), (iii) are the same as the corresponding hypotheses $H_{3}(\mathrm{i})$, (ii), (iii) and

(iv) for every $r>0$, there exist $\gamma_{r} \in L^{p^{\prime}}(T)$ and $c_{r}>0$ such that

$$
|F(t, x, y)| \leq \gamma_{r}(t)+c_{r}|y|^{p-1}
$$

for a.a. $t \in T$, all $|x| \leq r$, all $y \in \mathbb{R}^{N}$.

Now let $V=W_{0}^{1, p}$ for the Dirichlet problem, $V=W^{1, p}$ for the Neumann problem and $V=W_{\text {per }}^{1, p}=\left\{u \in W^{1, p}: u(0)=u(b)\right\}$ for the periodic problem. Then let $\widehat{\xi}: V \rightarrow V^{*}$ be defined by

$$
\langle\widehat{\xi}(u), h\rangle=\int_{0}^{b}\left|u^{\prime}\right|^{p-2}\left(u^{\prime}, h^{\prime}\right)_{\mathbb{R}^{N}} d t \quad \text { for all } u, h \in V .
$$

This map is continuous, monotone, thus maximal monotone too.

As before $\widehat{A}: L^{p} \rightarrow 2^{L^{p^{\prime}}}$ is the "lifting" of $A(\cdot)$ on the pair $\left(L^{p}, L^{p^{\prime}}\right)$ with $D(\widehat{A})=\{u \in$ $\left.L^{p}: S_{A(u(\cdot))}^{p^{\prime}} \neq \emptyset\right\}$. We know from Lemma 1 that $\widehat{A}$ is maximal monotone. Moreover, in the present setting with $D(A)=\mathbb{R}^{N}$, we have $C\left(T, \mathbb{R}^{N}\right) \subseteq D(\widehat{A})$. To see this note that the map $A(\cdot)$ has values in $P_{k c}\left(\mathbb{R}^{N}\right)$ and it is usc (see Gasiński-Papageorgiou [7], Proposition 3.2.14, p. 308). So, $A(\cdot)$ maps compact sets to compact sets (see Hu-Papageorgiou [11], Corollary 2.20 , p. 42). Let $u \in C\left(T, \mathbb{R}^{N}\right)$. Then

$$
|A(u(t))| \leq c_{13} \quad \text { for some } c_{13}>0, \text { all } t \in T .
$$

We can easily check that the multifunction $t \rightarrow A(u(t))$ has closed graph. Using the Yankov-von Neumann-Aumann selection theorem, we can find a measurable map $g: T \rightarrow$ $\mathbb{R}^{N}$ such that

$$
\begin{aligned}
& g(t) \in A(u(t)) \quad \text { for a.a. } t \in T, \\
\Rightarrow \quad & g \in L^{\infty} \subseteq L^{p^{\prime}} \quad(\text { see }(46)), \\
\Rightarrow \quad & S_{A(u(\cdot))}^{p^{\prime}} \neq \emptyset \quad \text { and so } u \in D(\widehat{A}) .
\end{aligned}
$$

Therefore $C\left(T, \mathbb{R}^{N}\right) \subseteq D(\widehat{A})$.

As before $N_{F}: V \rightarrow 2^{V^{*}}$ is defined by

$$
N_{F}(u)=S_{F\left(\cdot, u(\cdot), u^{\prime}(\cdot)\right)}^{p^{\prime}} \cdot
$$

From Sects. 3 and 4, we know that

$$
N_{F}(u) \in P_{w k c}\left(L^{p^{\prime}}\right) \quad\left(\text { convex case, hypotheses } H_{8}\right),
$$




$$
N_{F}(u) \in P_{f}\left(L^{p^{\prime}}\right) \quad\left(\text { nonconvex case, hypotheses } H_{9}\right) \text {. }
$$

We introduce the multivalued map $L: V \rightarrow 2^{V^{*}} \backslash\{\emptyset\}$ defined by

$$
L(u)=\widehat{\xi}(u)+\widehat{A}(u)+N_{F}(u) \text { for all } u \in V \subseteq D(\widehat{A}) .
$$

First we deal with the convex problem.

Proposition 8 If hypotheses $H_{6}, H_{7}, H_{8}$ hold, then $L(\cdot)$ is pseudomonotone.

Proof Evidently $L(\cdot)$ is bounded (that is, maps bounded sets to bounded sets). Consider sequences $\left\{u_{n}\right\}_{n \in \mathbb{N}} \subseteq V$ and $\left\{u_{n}^{*}\right\}_{n \in \mathbb{N}} \subseteq V^{*}$ such that

$$
\left\{\begin{array}{l}
u_{n} \stackrel{w}{\rightarrow} u \text { in } V, \quad u_{n}^{*} \stackrel{w}{\rightarrow} u^{*} \text { in } V^{*}, \quad\left(u_{n}, u_{n}^{*}\right) \in \operatorname{Gr} L \text { for all } n \in \mathbb{N}, \\
\lim \sup _{n \rightarrow+\infty}\left\langle u_{n}^{*}, u_{n}-u\right\rangle \leq 0 .
\end{array}\right.
$$

For every $n \in \mathbb{N}$, we have

$$
u_{n}^{*}=\widehat{\xi}\left(u_{n}\right)+g_{n}+f_{n} \quad \text { with } g_{n} \in \widehat{A}\left(u_{n}\right), f_{n} \in N_{F}\left(u_{n}\right) .
$$

Recall that $V \hookrightarrow C\left(T, \mathbb{R}^{N}\right)$ compactly. So, from (47) it follows that we can find $c_{14}>0$ such that

$$
\sup _{n \in \mathbb{N}}\left\|u_{n}\right\|_{C\left(T, \mathbb{R}^{N}\right)} \leq c_{14},
$$

$\Rightarrow \quad\left|A\left(u_{n}(t)\right)\right| \leq c_{15} \quad$ for some $c_{15}>0$, all $n \in \mathbb{N}$, all $t \in T$ (see hypotheses $H_{7}$ ),

$\Rightarrow\left\{g_{n}\right\}_{n \in \mathbb{N}} \subseteq L^{\infty} \subseteq L^{p^{\prime}}$ is bounded (see (47), (48) and hypothesis $H_{8}(\mathrm{iv})$ ).

So, we have

$$
\left\langle g_{n}, u_{n}-u\right\rangle=\left(g_{n}, u_{n}-u\right)_{p p^{\prime}} \rightarrow 0 \quad \text { and } \quad\left\langle f_{n}, u_{n}-u\right\rangle=\left(f_{n}, u_{n}-u\right)_{p p^{\prime}} \rightarrow 0
$$

From (47), (48), (49) it follows that

$$
\begin{aligned}
& \limsup _{n \rightarrow+\infty}\left\langle\widehat{\xi}\left(u_{n}\right), u_{n}-u\right\rangle \leq 0, \\
\Rightarrow \quad & \lim _{n \rightarrow+\infty}\left\langle\widehat{\xi}\left(u_{n}\right)-\widehat{\xi}(u), u_{n}-u\right\rangle=0 \quad \text { (since } \widehat{\xi}(\cdot) \text { is monotone). }
\end{aligned}
$$

For every $n \in \mathbb{N}$, we define

$$
\eta_{n}(t)=\left(a\left(u_{n}^{\prime}(t)\right)-a\left(u^{\prime}(t)\right), u_{n}^{\prime}(t)-u^{\prime}(t)\right)_{\mathbb{R}^{N}} .
$$

Evidently $\eta_{n} \in L^{1}(T)$ (Hölder's inequality), $\widehat{\eta}_{n} \geq 0$ (on account of the monotonicity of $\widehat{\xi}(\cdot))$ and from (50) we have

$$
\begin{aligned}
& \int_{0}^{b} \eta_{n}(t) d t \rightarrow 0, \\
\Rightarrow \quad & \eta_{n} \rightarrow 0 \text { in } L^{1}(T) .
\end{aligned}
$$


So, we may assume that

$$
\eta_{n}(t) \rightarrow 0 \text { for a.a. } t \in T, 0 \leq \eta_{n}(t) \leq \widehat{k}_{0}(t) \text { for a.a. } t \in T \text {, all } n \in \mathbb{N},
$$

with $\widehat{k}_{0} \in L^{1}(T)$. We have

$$
\begin{aligned}
\widehat{k}_{0}(t) \geq \eta_{n}(t) \geq & c_{0}\left[\left|u_{n}^{\prime}(t)\right|^{p}+\left|u^{\prime}(t)\right|^{p}\right] \\
& -c_{12}\left[1+\left|u_{n}^{\prime}(t)\right|^{p-1}\right]\left|u^{\prime}(t)\right|-c_{12}\left[1+\left|u^{\prime}(t)\right|^{p-1}\right]\left|u_{n}^{\prime}(t)\right| \\
& \left.\quad \text { (see hypotheses } H_{6}(\mathrm{i}),(\mathrm{ii})\right) .
\end{aligned}
$$

It follows that for a.a. $t \in T,\left\{u_{n}^{\prime}(t)\right\}_{n \in \mathbb{N}} \subseteq \mathbb{R}^{N}$ is bounded. Hence by passing to a subsequence (depending a priori on $t \in T$ ), we can say that

$$
u_{n}^{\prime}(t) \rightarrow v(t) \text { in } \mathbb{R}^{N} \text { as } n \rightarrow+\infty .
$$

From (51) in the limit as $n \rightarrow+\infty$, we obtain

$$
\begin{aligned}
& \left(a(v(t))-a\left(u^{\prime}(t)\right), v(t)-u^{\prime}(t)\right)_{\mathbb{R}^{N}=0,} \\
\Rightarrow \quad & u^{\prime}(t)=v(t) \quad(\text { since } a(\cdot) \text { is strictly monotone }) .
\end{aligned}
$$

So, for the initial sequence we have

$$
u_{n}^{\prime}(t) \rightarrow v(t) \text { in } \mathbb{R}^{N} \text { for a.a. } t \in T
$$

From (47) we have

$$
u_{n}^{\prime} \stackrel{w}{\rightarrow} u^{\prime} \text { in } L^{p}
$$

Then from (52) and (53) it follows that

$$
u_{n}^{\prime} \rightarrow u^{\prime} \text { in } L^{p}
$$

(by Vitali’s Theorem or see Gasiński-Papageorgiou [8], Problem 1.23, p. 38). Hence

$$
u_{n} \rightarrow v \text { in } V(\text { see }(47)) \text {. }
$$

We know that the sequences $\left\{g_{n}\right\}_{n \in \mathbb{N}},\left\{f_{n}\right\}_{n \in \mathbb{N}} \subseteq L^{p^{\prime}}$ are bounded. So, for at least a subsequence we have

$$
g_{n} \stackrel{w}{\rightarrow} g \text { and } f_{n} \stackrel{w}{\rightarrow} f \text { in } L^{p^{\prime}} \text { as } n \rightarrow+\infty
$$

We have

$$
\left(u_{n}, g_{n}\right) \in \mathrm{Gr} \widehat{A} \text { for all } n \in \mathbb{N} \text {. }
$$

Recall that $\widehat{A}$ is maximal monotone (see Lemma 1). Therefore from (54), (55), (56), we infer that

$$
(u, g) \in \mathrm{Gr} \widehat{A} .
$$


In addition, we have

$$
f_{n}(t) \in F\left(t, u_{n}(t), u_{n}^{\prime}(t)\right) \quad \text { for a.a. } t \in T \text {, all } n \in \mathbb{N} \text {. }
$$

From (54) we see that we may assume (by passing to a subsequence if necessary), that

$$
u_{n}(t) \rightarrow u(t) \text { and } u_{n}^{\prime}(t) \rightarrow u^{\prime}(t) \text { in } \mathbb{R}^{N} \text { for a.a. } t \in T
$$

Then (56), (58), (59) and hypothesis $H_{8}($ ii) imply that

$$
f(t) \in \overline{\mathrm{conv}} \limsup _{n \rightarrow+\infty} F\left(t, u_{n}(t), u_{n}^{\prime}(t)\right) \subseteq F\left(t, u(t), u^{\prime}(t)\right) \quad \text { for a.a. } t \in T .
$$

(see Hu-Papageorgiou [11], Proposition 3.9, p. 694),

$$
\Rightarrow \quad f \in N_{F}(u) \text {. }
$$

If in (48) we pass to the limit as $n \rightarrow+\infty$ and use (47), (54), (55) and the continuity of $\widehat{\xi}(\cdot)$, we obtain

$$
\begin{aligned}
u^{*} & =\widehat{\xi}(u)+g+f \quad \text { with } g \in \widehat{A}(u), f \in N_{F}(u)(\text { see (57), (60)), } \\
\Rightarrow \quad u^{*} & \in L(u) .
\end{aligned}
$$

Also, we have

$$
\left\langle u_{n}^{*}, u_{n}\right\rangle \rightarrow\left\langle u^{*}, u\right\rangle \quad(\operatorname{see}(47),(54)) .
$$

This proves that $L(\cdot)$ is pseudomonotone.

Proposition 9 If hypotheses $H_{6}, H_{7}, H_{8}$ hold, then $L(\cdot)$ is coercive, that is,

$$
\frac{\inf \left[\left\langle u^{*}, u\right\rangle: u^{*} \in L(u)\right]}{\|u\|} \rightarrow+\infty \quad \text { as }\|u\| \rightarrow+\infty .
$$

Proof On account of hypotheses $H_{8}$ (iii), (iv), given $\varepsilon>0$, we can find $\gamma_{\varepsilon} \in L^{p^{\prime}}(T)$ and $c_{\varepsilon}>0$ such that

$$
\sigma(t, x, y) \geq[\eta(t)-\varepsilon]|x|^{p}-\gamma_{\varepsilon}(t)-c_{\varepsilon}|y|^{p-1} \quad \text { for a.a. } t \in T \text {, all } x, y \in \mathbb{R}^{N} .
$$

Let $u^{*} \in L(u)$. We have

$$
\begin{gathered}
u^{*}=\widehat{\xi}(u)+g+f \quad \text { with } g \in \widehat{A}(u), f \in N_{F}(u), \\
\Rightarrow \quad\left\langle u^{*}, u\right\rangle=\int_{0}^{b}\left(a\left(u^{\prime}\right), u^{\prime}\right)_{\mathbb{R}^{N}} d t+\int_{0}^{b}(g(t), u(t))_{\mathbb{R}^{N}} d t+\int_{0}^{b}(f(t), u(t))_{\mathbb{R}^{N}} d t \\
\geq c_{0}\left\|u^{\prime}\right\|_{p}^{p}+\int_{0}^{b} \eta(t)|u|^{p} d t-\varepsilon\|u\|^{p}-c_{16}-c_{\varepsilon}\|u\|^{p-1} \\
\quad \text { with } c_{16}=\left\|\gamma_{\varepsilon}\right\|_{1}\left(\text { recall }(A(x), x)_{\mathbb{R}^{N}} \geq 0\right) \\
\geq\left[c_{17}-\varepsilon\right]\|u\|^{p}-c_{\varepsilon}\|u\|^{p-1}-c_{16} \quad \text { for some } c_{17}>0 .
\end{gathered}
$$


Choosing $\varepsilon \in\left(0, c_{17}\right)$, we infer that

$$
\begin{aligned}
& \left\langle u^{*}, u\right\rangle \geq c_{18}\|u\|^{p}-c_{\varepsilon}\|u\|^{p-1}-c_{16} \text { for some } c_{18}>0, \\
\Rightarrow & L(\cdot) \text { is coercive. }
\end{aligned}
$$

Now we are ready for the existence theorem for the convex problem.

Theorem 6 If hypotheses $H_{6}, H_{7}, H_{8}$ hold, then problem (1) admits a solution $\tilde{u} \in C^{1}$.

Proof Propositions 8 and 9 imply that $L(\cdot)$ is surjective. So, we can find $\tilde{u} \in V$ such that

$$
\begin{aligned}
& 0 \in L(\widetilde{u}), \\
\Rightarrow & \widehat{\xi}(\widetilde{u})+g+f=0 \quad \text { for some } g \in \widehat{A}(\widetilde{u}), f \in N_{F}(\widetilde{u}), \\
\Rightarrow & a\left(\widetilde{u}^{\prime}(t)\right)^{\prime}=g(t)+f(t) \quad \text { for a.a. } t \in T \text { (see Papageorgiou-Papalini [19]). }
\end{aligned}
$$

From (61) it follows that

$$
\begin{aligned}
& a\left(\tilde{u}^{\prime}(\cdot)\right) \in W^{1, p^{\prime}}, \\
\Rightarrow & \widetilde{u}^{\prime} \in C\left(T, \mathbb{R}^{N}\right), \\
\Rightarrow & \tilde{u} \in C^{1} \text { and solves (1). }
\end{aligned}
$$

Using hypotheses $H_{9}$ we can have an existence theorem for the nonconvex problem.

Theorem 7 If hypotheses $H_{6}, H_{7}, H_{9}$ hold, then problem (1) admits a solution $\tilde{u} \in C^{1}$.

Proof We consider the multifunction $N_{F}: V \rightarrow 2^{L^{p^{\prime}}}$ defined by

$$
N_{F}(u)=S_{F\left(\cdot, u(\cdot), u^{\prime}(\cdot)\right)}^{p^{\prime}} \quad \text { for all } u \in V
$$

From the proof of Theorem 4, we know that $N_{F}(\cdot)$ has decomposable values in $P_{f}\left(L^{p^{\prime}}\right)$ and it is 1sc. So, invoking Theorem 2, we can find a continuous map $e: V \rightarrow L^{p^{\prime}}$ such that

$$
e(u) \in N_{F}(u) \text { for all } u \in X .
$$

Then we consider the following boundary value problem

$$
\left\{\begin{array}{l}
a\left(u^{\prime}(t)\right)^{\prime} \in A(u(t))+e(u)(t) \quad \text { for a.a. } t \in T, \\
u \in B C .
\end{array}\right.
$$

Reasoning as in the proof of Theorem 6, we show that problem (62) admits a solution $\tilde{u} \in C^{1}$.

We can have relaxation theorems. First we will consider problem (35), but now our hypotheses on $a(\cdot)$ are less restrictive and so we can incorporate in our analysis more differential operators.

So, we introduce the following hypotheses on $a(\cdot)$ and $F(\cdot, \cdot)$ : 
$H_{10}: a: \mathbb{R}^{N} \rightarrow \mathbb{R}^{N}$ is a map such that $a(0)=0$ and

(i) $a(\cdot)$ is continuous and strictly monotone;

(ii) for every $r>0$, there exists $\widehat{c}_{r}>0$ such that

$$
\widehat{c}_{r}\left|y-y^{\prime}\right|^{2} \leq\left(a(y)-a\left(y^{\prime}\right), y-y^{\prime}\right)_{\mathbb{R}^{N}} \text { for all }|y|,\left|y^{\prime}\right| \leq r
$$

(iii) $|a(y)| \leq c_{19}\left[1+|y|^{p-1}\right]$ for all $y \in \mathbb{R}^{N}$, some $c_{19}>0$;

(iv) $c_{0}|y|^{p} \leq(a(y), y)_{\mathbb{R}^{N}}$ for all $y \in \mathbb{R}^{N}$, some $c_{0}>0$ and $1<p<+\infty$.

Remark 8 Note that hypothesis $H_{10}$ (ii) is weaker than the corresponding hypothesis $H_{4}(\mathrm{ii})$, since now the strong monotonicity condition is local. This fits in the present framework more differential operators (see the examples below).

Example 3 The following maps satisfy hypotheses $H_{10}$ :

(a) $a(y)=|y|^{p-2} y, 1<p \leq 2$.

(b) $a(y)=|y|^{p-2} y+|y|^{q-2} y, 1<q<p<+\infty, q \leq 2$.

(c) $a(y)=\left[1+|y|^{2}\right]^{\frac{p-2}{2}} y, 1<p<+\infty$.

$H_{11}: F: T \times \mathbb{R}^{N} \rightarrow P_{k}\left(\mathbb{R}^{N}\right)$ is a multifunction such that

(i) for every $x \in \mathbb{R}^{N}, t \rightarrow F(t, x)$ is graph measurable;

(ii) for every $r>0$, there exists $k_{r} \in L^{1}(T)$ such that

$$
h(F(t, x), F(t, v)) \leq k_{r}(t)|x-v| \text { for a.a. } t \in T \text {, all }|x|,|v| \leq r,
$$

and $b\left\|k_{r}\right\|_{1}<\widehat{c_{r}}$

(iii) if $\sigma_{0}(t, x)=\inf \left[(v, x)_{\mathbb{R}^{N}}: v \in F(t, x)\right]$, then

$$
\liminf _{|x| \rightarrow+\infty} \frac{\sigma_{0}(t, x)}{|x|^{p}} \geq \vartheta(t) \quad \text { uniformly for a.a. } t \in T,
$$

with $\vartheta \in L^{\infty}(T), \vartheta(t) \geq-c_{0} \widehat{\lambda}_{1}$ for a.a. $t \in T, \vartheta \not \equiv-c_{0} \widehat{\lambda}_{1}$;

(iv) for every $M>0$, there exists $\gamma_{M} \in L^{p^{\prime}}(T)$ such that

$$
|F(t, x)| \leq \gamma_{M}(t) \quad \text { for a.a. } t \in T \text {, all }|x| \leq M .
$$

Theorem 8 If hypotheses $H_{10}, H_{7}, H_{11}$ hold, then for problem (35), $S_{c}=\bar{S}^{C^{1}}$.

Proof Evidently $S_{c}$ is closed in $C^{1}$. Also, keeping the notation introduced in the proof of Theorem 5, we have that

$$
\begin{aligned}
& \left\{v_{n}\right\}_{n \in \mathbb{N}} \subseteq W^{1, p} \text { is bounded, } \\
& v_{n} \rightarrow u \text { in } W^{1,2} \text { as } n \rightarrow+\infty
\end{aligned}
$$

(recall $u \in S_{c}$, see the proof of Theorem 5). We have

$$
\left\{\begin{array}{l}
a\left(v_{n}^{\prime}(t)\right)^{\prime} \in A\left(v_{n}(t)\right)+\beta_{n}\left(v_{n}\right)(t) \quad \text { for a.a. } t \in T, \\
v_{n}(0)=v_{n}(b)=0
\end{array}\right.
$$


From (63) and since $W^{1, p} \hookrightarrow C\left(T, \mathbb{R}^{N}\right)$ compactly, we have

$$
\left|A\left(v_{n}(t)\right)\right| \leq c_{20} \quad \text { for some } c_{20}>0 \text {, all } t \in T \text {, all } n \in \mathbb{N} \text { (see hypotheses } H_{7} \text { ). }
$$

Also from (63) and hypothesis $H_{11}(\mathrm{iv})$, we have that

$$
\left\{\beta_{n}\left(v_{n}\right)\right\}_{n \in \mathbb{N}} \subseteq L^{p^{\prime}} \text { is bounded. }
$$

Then, from (65), (66) and (67) it follows that

$$
\left\{a\left(v_{n}^{\prime}\right)^{\prime}\right\}_{n \in \mathbb{N}} \subseteq L^{p^{\prime}} \text { is bounded. }
$$

From (68) and reasoning as in the proof of Proposition 5, we obtain that

$$
\left\{v_{n}\right\}_{n \in \mathbb{N}} \subseteq C^{1} \text { is relatively compact. }
$$

On account of (64), we have

$$
\begin{aligned}
v_{n} & \rightarrow u \text { in } C^{1} \text { with } v_{n} \in S(\text { see }(65)), \\
\Rightarrow \quad S_{c} & =\bar{S}^{C^{1}} .
\end{aligned}
$$

If we strengthen the conditions on $A(\cdot)$, we can have relaxation for the case where $F$ is also dependent on $u^{\prime}$ and the boundary condition is always Dirichlet.

So, the new conditions on the data are:

$H_{12}: A: \mathbb{R}^{N} \rightarrow \mathbb{R}^{N}$ is locally Lipschitz and strictly monotone.

$H_{13}: F: T \times \mathbb{R}^{N} \times \mathbb{R}^{N} \rightarrow P_{k}\left(\mathbb{R}^{N}\right)$ is a multifunction such that

(i) for all $x, y \in \mathbb{R}^{N}, t \rightarrow F(t, x, y)$ is graph measurable;

(ii) for every $r>0$, there exists $k_{r} \in L^{1}(T)$ such that

$$
h\left(F(t, x, y), F\left(t, x^{\prime}, y^{\prime}\right)\right) \leq k_{r}(t)\left[\left|x-x^{\prime}\right|+\left|y-y^{\prime}\right|\right]
$$

for a.a. $t \in T$, all $|x|,\left|x^{\prime}\right|,|y|,\left|y^{\prime}\right| \leq r$

(iii) if $\sigma(t, x, y)=\inf [(v, x): v \in F(t, x, y)]$, then

$$
\liminf _{|x| \rightarrow+\infty} \frac{\sigma(t, x, y)}{|x|^{p}} \geq \vartheta(t) \quad \text { uniformly for a.a. } t \in T \text {, all } y \in \mathbb{R}^{N},
$$

with $\vartheta \in L^{\infty}(T), \vartheta(t) \geq-c_{0} \widehat{\lambda}_{1}$ for a.a. $t \in T, \vartheta \neq \equiv-c_{0} \widehat{\lambda}_{1}$;

(iv) for every $r>0$, there exist $\gamma_{r} \in L^{p^{\prime}}(T)$ and $c_{r}>0$ such that

$$
|F(t, x, y)| \leq \gamma_{r}(t)+c_{r}|y|^{p-1} \quad \text { for a.a. } t \in T \text {, all }|x| \leq r \text { and all } y \in \mathbb{R}^{N} \text {. }
$$

Now $S_{c}$ (resp. $S$ ) is the solution set of the convex (resp. nonconvex) problem (1) with Dirichlet boundary condition.

Theorem 9 If hypotheses $H_{10}, H_{12}, H_{13}$ hold, then $S_{c}=\bar{S}^{C^{1}}$. 
Proof Let $u \in S_{c}$. We have

$$
\left\{\begin{array}{l}
a\left(u^{\prime}(t)\right)=A(u(t))+f(t) \quad \text { for a.a. } t \in T, \\
u(0)=u(b)=0,
\end{array}\right.
$$

with $f \in S_{\overline{\operatorname{conv}} F\left(\cdot, u(\cdot), u^{\prime}(\cdot)\right)}^{p^{\prime}}$.

Invoking Proposition 3.30, p. 185, of Hu-Papageorgiou [11], we can find $\left\{f_{n}\right\}_{n \in \mathbb{N}} \subseteq$ $S_{F\left(\cdot, u(\cdot), u^{\prime}(\cdot)\right)}^{p}$ such that

$$
f_{n} \stackrel{w}{\rightarrow} f \text { in } L^{p^{\prime}}
$$

Let $\varepsilon_{n} \rightarrow 0^{+}$and $y \in W^{1, p}$. We introduce the multifunction $\widehat{L}_{n}: T \rightarrow 2^{\mathbb{R}^{N}}$ defined by

$$
\widehat{L}_{n}(t)=\left\{v \in \mathbb{R}^{n}:\left|f_{n}(t)-v\right|<\varepsilon_{n}+d\left(f_{n}(t), F\left(t, y(t), y^{\prime}(t)\right)\right), v \in F\left(t, y(t), y^{\prime}(t)\right)\right\}
$$

for all $n \in \mathbb{N}$, all $t \in T$.

By modifying $\widehat{L}_{n}(\cdot)$ on a Lebesgue-null set if necessary, we can say that

$$
\widehat{L}_{n}(t) \neq \emptyset \quad \text { for all } t \in T \text {, all } n \in \mathbb{N} \text {. }
$$

Hypotheses $H_{13}(\mathrm{i})$, (ii) imply that $t \rightarrow F\left(t, y(t), y^{\prime}(t)\right)$ is graph measurable and so

$$
\operatorname{Gr} \widehat{L} \in \mathcal{L}_{T} \otimes B\left(\mathbb{R}^{N}\right) \otimes B\left(\mathbb{R}^{N}\right)
$$

Then the Yankov-von Neumann-Aumann selection theorem implies that we can find $h_{n} \in$ $L^{p^{\prime}}$ such that

$$
h_{n}(t) \in L_{n}(t) \quad \text { for a.a. } t \in T \text {, all } n \in \mathbb{N} \text {. }
$$

Hence, if we consider the multifunction $\Gamma_{n}: V \rightarrow 2^{L^{p^{\prime}}}, n \in \mathbb{N}$, defined by

$$
\Gamma_{n}(y)=\left\{h \in S_{F\left(\cdot, y(\cdot), y^{\prime}(\cdot)\right)}^{p^{\prime}}:\left|f_{n}(t)-h(t)\right|<\varepsilon_{n}+d\left(f_{n}(t), F\left(t, y(t), y^{\prime}(t)\right)\right) \text { for a.a. } t \in T\right\},
$$

then from the previous argument we infer that $\Gamma_{n}(y) \neq \emptyset$, for all $y \in V$, all $n \in \mathbb{N}$. Moreover, on account of Lemma 8.3, p. 239, of Hu-Papageorgiou [11], we have that

$$
y \rightarrow \overline{\Gamma_{n}(y)} \|^{\|\cdot\|_{p^{\prime}}} \text { is lsc for all } n \in \mathbb{N} .
$$

In addition this multifunction has decomposable values. So, we can apply Theorem 2 and obtain a continuous map $\widehat{\gamma}_{n}: V \rightarrow L^{p^{\prime}}, n \in \mathbb{N}$, such that

$$
\widehat{\gamma}_{n}(y) \in \overline{\Gamma_{n}(y)} \|^{\|\cdot\|_{p^{\prime}}} \text { for all } y \in V \text {, all } n \in \mathbb{N} .
$$

Then we consider the following boundary value problem

$$
\left\{\begin{array}{l}
a\left(v^{\prime}(t)\right)^{\prime}=A(v(t))+\widehat{\gamma}_{n}(v)(t) \quad \text { for a.a. } t \in T, \\
v(0)=v(b)=0, n \in \mathbb{N} .
\end{array}\right.
$$

This problem has a solution $v_{n} \in C^{1}, n \in \mathbb{N}$. 
We have

$$
\begin{gathered}
-\int_{0}^{b}\left(a\left(v_{n}^{\prime}\right)^{\prime}, v_{n}\right)_{\mathbb{R}^{N}} d t \leq \int_{0}^{b}\left(\widehat{\gamma}_{n}\left(v_{n}\right),-v_{n}\right)_{\mathbb{R}^{N}} d t \\
\left(\text { recall that }(A(x), x)_{\mathbb{R}^{N}} \geq 0 \text { for all } x \in \mathbb{R}^{N}\right), \\
\Rightarrow \quad c_{0}\left\|v_{n}^{\prime}\right\|_{p}^{p} \leq \int_{0}^{b}\left(\widehat{\gamma}_{n}\left(v_{n}\right),-v_{n}\right)_{\mathbb{R}^{N}} d t
\end{gathered}
$$

(performing integration by parts and using $H_{10}$ (iv)) .

From hypotheses $H_{13}$ (iii), (iv) given $\varepsilon>0$, we can find $k_{\varepsilon} \in L^{p^{\prime}}(T)$ such that

$$
\sigma(t, x, y) \geq(\vartheta(t)-\varepsilon)|x|^{p}-k_{\varepsilon}(t) \quad \text { for a.a. } t \in T \text {, all } x, y \in \mathbb{R}^{N} .
$$

Using this in (70), we obtain

$$
\begin{aligned}
& c_{0}\left\|v_{n}^{\prime}\right\|_{p}^{p}+\int_{0}^{b} \vartheta(t)\left|v_{n}\right|^{p} d t-\varepsilon\left\|v_{n}\right\|^{p} \leq c_{21} \text { for some } c_{21}>0, \text { all } n \in \mathbb{N}, \\
\Rightarrow & \left\{v_{n}\right\}_{n \in \mathbb{N}} \subseteq W^{1, p} \text { is bounded (as before choosing } \varepsilon>0 \text { and using } H_{13}(\text { iii)). }
\end{aligned}
$$

Then from (71) as in the proof of Proposition 5 we infer that

$$
\left\{v_{n}\right\}_{n \in \mathbb{N}} \subseteq C^{1} \text { is relatively compact. }
$$

So, we may assume that

$$
v_{n} \rightarrow v \text { in } C^{1} .
$$

We have

$$
\begin{aligned}
& a\left(u^{\prime}(t)\right)^{\prime}-a\left(v_{n}^{\prime}(t)\right)^{\prime} \\
= & A(u(t))-A\left(v_{n}(t)\right)+f(t)-\widehat{\gamma}_{n}\left(v_{n}\right)(t) \\
\leq & c_{22}\left|u(t)-v_{n}(t)\right|+f(t)-\widehat{\gamma}_{n}\left(v_{n}\right)(t)
\end{aligned}
$$

for a.a. $t \in T$, all $n \in \mathbb{N}$ and some $c_{22}>0$,

$\Rightarrow \quad\left|a\left(u^{\prime}(t)\right)-a\left(v_{n}^{\prime}(t)\right) \leq c_{22} \int_{0}^{t}\right| u(s)-v_{n}(s)|d s+| \int_{0}^{t}\left(f(s)-\widehat{\gamma}_{n}\left(v_{n}\right)(s)\right) d s \mid$

for all $n \in \mathbb{N}$ (using the $B C$ ).

Note that

$$
\begin{aligned}
& \left|\int_{0}^{t}\left(f(s)-\widehat{\gamma}_{n}\left(v_{n}\right)(s)\right) d s\right| \\
\leq & \left|\int_{0}^{t}\left(f(s)-f_{n}(s)\right) d s\right|+\left|\int_{0}^{t}\left(f_{n}(s)-\widehat{\gamma}_{n}\left(v_{n}\right)(s)\right) d s\right| \\
\leq & \left|\int_{0}^{t}\left(f(s)-f_{n}(s)\right) d s\right|+\int_{0}^{t}\left|f_{n}(s)-\widehat{\gamma}_{n}\left(v_{n}\right)(s)\right| d s
\end{aligned}
$$




$$
\leq\left|\int_{0}^{t}\left(f(s)-f_{n}(s)\right) d s\right|+\varepsilon_{n} b+\int_{0}^{t} h\left(F\left(s, u(s), u^{\prime}(s)\right), F\left(s, v_{n}(s), v_{n}^{\prime}(s)\right)\right) d s .
$$

We have

$$
\varepsilon_{n}^{\prime}(t)=\left|\int_{0}^{t}\left(f(s)-f_{n}(s)\right) d s\right|+\varepsilon_{n} b \rightarrow 0 \quad \text { for all } t \in T \text {, as } n \rightarrow+\infty \text { (see (69)). }
$$

We return to (73) and use (74), (75) and hypotheses $H_{10}$ (ii) and $H_{13}($ ii). We obtain

$$
\begin{aligned}
c_{23}\left|u^{\prime}(t)-v_{n}^{\prime}(t)\right| \leq \varepsilon_{n}^{\prime}(t)+ & \int_{0}^{t} \int_{0}^{s}\left|u^{\prime}(\tau)-v_{n}^{\prime}(\tau)\right| d \tau d s \\
& +\int_{0}^{t} k_{0}(s)\left[\int_{0}^{s}\left|u(\tau)-v_{n}(\tau)\right| d \tau+\left|u^{\prime}(s)-v_{n}^{\prime}(s)\right|\right] d s \\
& \quad \text { for some } c_{23}>0, k_{0} \in L^{1}(T) \text { and all } n \in \mathbb{N} .
\end{aligned}
$$

Passing to the limit as $n \rightarrow+\infty$ and using (72) and (75), we obtain

$$
c_{23}\left|u^{\prime}(t)-v^{\prime}(t)\right| \leq \int_{0}^{t} k(s)\left|u^{\prime}(s)-v^{\prime}(s)\right| d s+\int_{0}^{t}(b k(s)+1) \int_{0}^{s}\left|u^{\prime}(\tau)-v^{\prime}(\tau)\right| d \tau d s
$$

for all $t \in T$.

Then Proposition 1.7.87, p. 128, of Denkowski-Migorski-Papageorgiou [3], implies that

$$
\begin{aligned}
& u^{\prime}(t)=v^{\prime}(t) \quad \text { for all } t \in T, \\
\Rightarrow \quad & u=v+\eta \quad \text { with } \eta \in \mathbb{R}^{N} .
\end{aligned}
$$

The Dirichlet boundary condition implies $\eta=0$. We have

$$
\begin{aligned}
v_{n} & \rightarrow u \text { in } C^{1} \text { and } v_{n} \in S \text { for all } n \in \mathbb{N}, \\
\Rightarrow \quad S_{c} & =\bar{S}^{C^{1}} .
\end{aligned}
$$

\section{Applications}

First we present an application on differential variational inequalities.

So, let $\mathbb{R}_{+}^{N}$ be the positive cone of $\mathbb{R}^{N}$. We consider the indicator function of this cone, namely the function

$$
i_{\mathbb{R}_{+}^{N}}(x)= \begin{cases}0 & \text { if } x \in \mathbb{R}_{+}^{N} \\ +\infty & \text { if } x \notin \mathbb{R}_{+}^{N}\end{cases}
$$

This function is convex and lower semicontinuous. We consider the subdifferential in the sense of convex analysis $\partial i_{\mathbb{R}_{+}^{N}}(x)$. We know that for all $x=\left(x_{k}\right)_{k=1}^{N} \in \mathbb{R}_{+}^{N}$ we have

$$
\partial i_{\mathbb{R}_{+}^{N}}(x)=N_{\mathbb{R}_{+}^{N}}(x)= \begin{cases}0 & \text { if } x_{k}>0 \text { for all } k \in\{1, \ldots, N\}, \\ -\mathbb{R}_{+}^{N} \cap\{x\}^{\perp} & \text { if } x_{k}=0 \text { for some } k \in\{1, \ldots, N\},\end{cases}
$$


and $\partial i_{\mathbb{R}_{+}^{N}}(x)=\emptyset$ if $x \notin \mathbb{R}_{+}^{N}$ (see Gasiński-Papageorgiou [7], p. 526). The set $N_{\mathbb{R}_{+}^{N}}(x)$ is known as the normal cone to $\mathbb{R}_{+}^{N}$ at $x$. We set

$$
A(x)=\partial i_{\mathbb{R}_{+}^{N}}(x)=N_{\mathbb{R}_{+}^{N}}(x) .
$$

Evidently $A(\cdot)$ is maximal monotone, $0=A(0)$ and $D(A) \neq \mathbb{R}^{N}$. Given $u=\left(u_{k}\right)_{k=1}^{N} \in$ $W^{1, p}$, we introduce the following sets

$$
\begin{aligned}
& T_{+}(u)=\left\{t \in T: u_{k}(t)>0 \text { for all } k \in\{1, \ldots, N\}\right\}, \\
& T_{0}(u)=\left\{t \in T: u_{k}(t)=0 \text { for some } k \in\{1, \ldots, N\}\right\} .
\end{aligned}
$$

We consider the problem

$$
\left\{\begin{array}{l}
a\left(u^{\prime}(t)\right)^{\prime} \in A(u(t))+F\left(t, u(t), u^{\prime}(t)\right) \text { for a.a. } t \in T, \\
u \in B C,
\end{array}\right.
$$

with $A(\cdot)$ as above and $a: \mathbb{R}^{N} \rightarrow \mathbb{R}^{N}, F: T \times \mathbb{R}^{N} \times \mathbb{R}^{N} \rightarrow P_{k c}\left(\mathbb{R}^{N}\right)$ satisfying hypotheses $H_{0}$ and $H_{2}$ respectively.

Problem (76) is equivalent to the following differential variational inequality

$$
\left\{\begin{array}{l}
a\left(u^{\prime}(t)\right)^{\prime} \in F\left(t, u(t), u^{\prime}(t)\right) \quad \text { for a.a. } t \in T_{+}(u), \\
a\left(u^{\prime}(t)\right)^{\prime} \in F\left(t, u(t), u^{\prime}(t)\right)-g(t) \quad \text { for a.a. } t \in T_{0}(u), \\
u(t), g(t) \in \mathbb{R}_{+}^{N}, \quad(g(t), u(t))_{\mathbb{R}^{N}}=0 \quad \text { for a.a. } t \in T, \\
u \in B C, 2 \leq p .
\end{array}\right.
$$

On account of Theorem 3, problem (77) has a solution $\tilde{u} \in C^{1}$.

Next we consider the following optimal control problem

$$
J(u, v)=\int_{0}^{b} L\left(t, u(t), u^{\prime}(t), v^{\prime}(t)\right) d t \rightarrow \inf =m,
$$

subject to:

$$
\left\{\begin{array}{l}
a\left(u^{\prime}(t)\right)^{\prime} \in A(u(t))+f(t, u(t)) u^{\prime}(t)+B(t) v(t) \quad \text { for a.a. } t \in T, \\
u \in B C, v \in S_{K(\cdot, u(\cdot))}^{1} .
\end{array}\right.
$$

Here $u(\cdot)$ is the state of the system and $v(\cdot)$ the control function. So, problem (78) has second order dynamics and a priori feedback since the control constraint multifunction $K$ is state dependent.

The hypotheses on the data of this problem are the following:

$H_{14}: f: T \times \mathbb{R}^{N} \rightarrow \mathcal{L}\left(\mathbb{R}^{N}, \mathbb{R}^{N}\right)$ is a function such that

(i) for all $x, y \in \mathbb{R}^{N}, t \rightarrow f(t, x) y$ is measurable;

(ii) for a.a. $t \in T, x \rightarrow f(t, x)$ is continuous;

(iii)

$$
\liminf _{|x| \rightarrow+\infty} \frac{(f(t, x) y, x)_{\mathbb{R}^{N}}}{|x|^{p}} \geq \vartheta(t) \text { uniformly for a.a. } t \in T \text {, all } y \in \mathbb{R}^{N},
$$

with $\vartheta \in L^{\infty}(T), \vartheta(t) \geq-c_{0} \widehat{\lambda}_{1}$ for a.a. $t \in T, \vartheta \not \equiv-c_{0} \widehat{\lambda}_{1}$; 
(iv) for every $r>0$, there exists $c_{r}>0$ such that

$$
\|f(t, x)\|_{\mathcal{L}} \leq c_{r} \text { for a.a. } t \in T \text {, all }|x| \leq r
$$

$H_{15}: B \in L^{p^{\prime}}\left(T, \mathcal{L}\left(\mathbb{R}^{m}, \mathbb{R}^{N}\right)\right)$.

$H_{16}: K: T \times \mathbb{R}^{N} \rightarrow P_{k c}\left(\mathbb{R}^{m}\right)$ is a multifunction such that

(i) $(t, x) \rightarrow K(t, x)$ is measurable;

(ii) for a.a. $t \in T, x \rightarrow K(t, x)$ is closed;

(iii) $|K(t, x)| \leq M$ for a.a. $t \in T$, all $x \in \mathbb{R}^{N}$ with $M>0$.

$H_{17}: L: T \times \mathbb{R}^{N} \times \mathbb{R}^{N} \times \mathbb{R}^{m} \rightarrow \overline{\mathbb{R}}=\mathbb{R} \cup\{+\infty\}$ is a measurable integrand such that

(i) for a.a. $t \in T,(x, y, v) \rightarrow L(t, x, y, v)$ is lsc;

(ii) for a.a. $t \in T$ and all $x \in \mathbb{R}^{N},(y, v) \rightarrow L(t, x, y, v)$ is convex;

(iii) $\eta(t)-c_{24}(|x|+|y|+|v|) \leq L(t, x, y, v)$ for a.a. $t \in T$, all $x, y \in \mathbb{R}^{N}, v \in \mathbb{R}^{m}$ and with $\eta \in L^{1}(T)$.

On account of hypothesis $H_{16}(\mathrm{i})$ we can find $k_{n}: T \times \mathbb{R}^{N} \rightarrow \mathbb{R}^{m}, n \in \mathbb{N}$, measurable functions such that

$$
K(t, x)={\overline{\left\{k_{n}(t, x)\right.}}_{n \in \mathbb{N}}
$$

(see Hu-Papageorgiou [11], Theorem 2.4, p. 156). Then

$$
\begin{aligned}
& G(t, x)=B(t) K(t, x)={\overline{\left\{B(t) k_{n}(t, x)\right\}_{n \in \mathbb{N}}}}_{\Rightarrow}, \\
\Rightarrow & G(\cdot, \cdot) \text { is measurable. }
\end{aligned}
$$

Also, hypotheses $H_{14}(\mathrm{i})$, (ii) imply that $f$ is jointly measurable (see PapageorgiouWinkert [20], Theorem 2.2.31, p. 106). Therefore

$$
F(t, x, y)=f(t, x) y+G(t, x)
$$

is a measurable multifunction. A straightforward application of the Yankov-von NeumannAumann selection shows that the dynamic constraint of the optimal control problem is equivalent to the following multivalued boundary value problem

$$
\left\{\begin{array}{l}
a\left(u^{\prime}(t)\right)^{\prime} \in A(u(t))+F\left(t, u(t), u^{\prime}(t)\right) \quad \text { for a.a. } t \in T, \\
u \in B C .
\end{array}\right.
$$

On account of hypotheses $H_{14}, H_{15}, H_{16}$, the multifunction $F(t, x, y)$ satisfies hypotheses $\mathrm{H}_{2}$.

Moreover, hypotheses $H_{17}$ imply that the cost functional $J(\cdot, \cdot)$ is sequentially lower semicontinuous on $L^{p} \times L_{w}^{p} \times L_{w}^{p}$ (see Papageorgiou-Winkert [20], Theorem 5.6.55, p. 458).

Finally we assume that $a(\cdot)$ and $A(\cdot)$ satisfy hypotheses $H_{0}$ and $H_{1}$ respectively.

Let $\left\{\left(u_{n}, v_{n}\right)\right\}_{n \in \mathbb{N}} \subseteq C^{1} \times L^{1}$ be a minimizing sequence for problem (78). Then we can show that $\left\{u_{n}\right\}_{n \in \mathbb{N}} \subseteq W^{1, p}$ is bounded. So, we may assume that

$$
u_{n} \stackrel{w}{\rightarrow} u \text { in } W^{1, p} \text { and } u_{n} \rightarrow u \text { in } C\left(T, \mathbb{R}^{N}\right)
$$


Note that

$$
f\left(t, u_{n}\right) u_{n}^{\prime} \stackrel{w}{\rightarrow} f(t, u) u^{\prime} \quad \text { in } L^{p^{\prime}} \text { (see hypothesis } H_{14}(\text { ii) and (79)). }
$$

On account of hypothesis $H_{16}$ (iii) we may assume that

$$
v_{n} \stackrel{w}{\rightarrow} v \text { in } L^{p^{\prime}} \text { and } v(t) \in K(t, u(t)) \text { for a.a. } t \in T .
$$

Therefore the state-control pair $(u, v)$ is admissible. Also, we have

$$
\begin{aligned}
J(u, v) & \leq \liminf _{n \rightarrow+\infty} J\left(u_{n}, v_{n}\right)=m, \\
\Rightarrow \quad J(u, v) & =m .
\end{aligned}
$$

So, the optimal control problem (78) has a solution.

Finally consider the following control system

$$
\left\{\begin{array}{l}
a\left(u^{\prime}(t)\right)^{\prime} \in \nabla \varphi(u(t))+f\left(t, u(t), u^{\prime}(t)\right)+B(t) v(t) \quad \text { for a.a. } t \in T \\
u(0)=u(b)=0, \quad v(t) \in K(t, u(t)) \text { for a.a. } t \in T
\end{array}\right.
$$

Now we assume the following:

$H_{18}: \varphi \in C^{1}\left(\mathbb{R}^{N}\right)$ and $x \rightarrow \nabla \varphi(x)$ is locally Lipschitz, strictly monotone.

$H_{19}: f: T \times \mathbb{R}^{N} \times \mathbb{R}^{N} \rightarrow \mathbb{R}^{N}$ is a function such that

(i) for all $x, y \in \mathbb{R}^{N}, t \rightarrow f(t, x, y)$ is measurable;

(ii) for a.a. $t \in T, f(t, \cdot, \cdot)$ is $l(t)$-Lipschitz with $l \in L^{1}(T)$;

(iii)

$$
\liminf _{|x| \rightarrow+\infty} \frac{(f(t, x, y), x)_{\mathbb{R}^{N}}}{|x|^{p}} \geq \vartheta(t) \text { uniformly for a.a. } t \in T \text {, all } y \in \mathbb{R}^{N},
$$

with $\vartheta \in L^{\infty}(T), \vartheta(t) \geq-c_{0} \widehat{\lambda}_{1}$ for a.a. $t \in T, \vartheta \not \equiv-c_{0} \widehat{\lambda}_{1}$;

(iv) for every $r>0$, there exist $k_{r} \in L^{p^{\prime}}$ and $c_{r}>0$ such that

$$
|f(t, x, y)| \leq k_{r}(t)+c_{r}|y|^{p-1} \text { for a.a. } t \in T \text {, all }|x| \leq r \text {, all } y \in \mathbb{R}^{N} .
$$

$H_{20}: K: T \times \mathbb{R}^{N} \rightarrow P_{k}\left(\mathbb{R}^{m}\right)$ is a multifunction such that

(i) $t \rightarrow K(t, x)$ is graph measurable;

(ii) $h\left(K(t, x), K\left(t, x^{\prime}\right)\right) \leq \widehat{l}(t)\left|x-x^{\prime}\right|$ for a.a. $t \in T$, all $x, x^{\prime} \in \mathbb{R}^{N}$, with $\widehat{l} \in L^{1}(T)$;

(iii) $|K(t, x)| \leq M$ for a.a. $t \in T$, all $x \in \mathbb{R}^{N}$, some $M>0$.

We denote by $P$ (resp. $P_{c}$ ) the set of states generated by controls in $K$ (resp. $\overline{c o n v} K$ ). Then assuming $H_{10}, H_{18}, H_{15}, H_{19}$ and $H_{20}$ and using Theorem 9, we conclude that

$$
P_{c}=\bar{P}^{C^{1}} \text {. }
$$

This result can lead to admissible relaxation methods for optimal control problems (see Papageorgiou-Rădulescu-Repovš [23] and Papageorgiou-Vetro-Vetro [24], who deal with first order systems). 
Funding Note Open access funding provided by Università degli Studi di Palermo within the CRUI-CARE Agreement.

Open Access This article is licensed under a Creative Commons Attribution 4.0 International License, which permits use, sharing, adaptation, distribution and reproduction in any medium or format, as long as you give appropriate credit to the original author(s) and the source, provide a link to the Creative Commons licence, and indicate if changes were made. The images or other third party material in this article are included in the article's Creative Commons licence, unless indicated otherwise in a credit line to the material. If material is not included in the article's Creative Commons licence and your intended use is not permitted by statutory regulation or exceeds the permitted use, you will need to obtain permission directly from the copyright holder. To view a copy of this licence, visit http://creativecommons.org/licenses/by/4.0/.

\section{References}

1. Bader, R.: A topological fixed point index theory for evolution inclusions. Z. Anal. Anwend. 20, 3-15 (2001)

2. Bressan, A., Colombo, G.: Extensions and selections of maps with decomposable values. Stud. Math. 90, 69-86 (1988)

3. Denkowski, Z., Migorski, S., Papageorgiou, N.S.: An Introduction to Nonlinear Analysis: Applications. Kluwer Academic, Boston (2003)

4. Frigon, M., Montoki, E.: Systems of differential inclusions with maximal monotone terms. J. Math. Anal. Appl. 323, 1134-1151 (2006)

5. Fryszkowski, A.: Continuous selections for a class of non-convex multivalued maps. Stud. Math. 76, 163-174 (1983)

6. Fryszkowski, A.: Fixed Point Theory for Decomposable Sets. Kluwer Academic, New York (2005)

7. Gasiński, L., Papageorgiou, N.S.: Nonlinear Analysis. Ser. Math. Anal. Appl., vol. 9. CRC Press Boca Raton (2006)

8. Gasiński, L., Papageorgiou, N.S.: Exercises in Analysis. Part 2. Springer, Switzerland (2016)

9. Gasiński, L., Papageorgiou, N.S.: Nonlinear multivalued periodic systems. J. Dyn. Control Syst. 25, 219-243 (2019)

10. Halidias, N., Papageorgiou, N.S.: Existence and relaxation results for nonlinear second-order multivalued boundary value problems in $R^{N}$. J. Differ. Equ. 147, 123-154 (1998)

11. Hu, S., Papageorgiou, N.S.: Handbook of Multivalued Analysis. Vol. I: Theory. Kluwer Academic, Dordrecht (1997)

12. Kandilakis, D., Papageorgiou, N.S.: Existence theorems for nonlinear boundary value problems for second order differential inclusions. J. Differ. Equ. 132, 107-125 (1996)

13. Kyritsi, S., Matzakos, N., Papageorgiou, N.S.: Periodic problems for strongly nonlinear second-order differential inclusions. J. Differ. Equ. 183, 279-302 (2002)

14. Leoni, G.: A First Course in Sobolev Spaces. Graduate Studies in Math., vol. 105. Am. Math. Soc., Providence (2009)

15. Ma, H., Xue, X.: Second order nonlinear multivalued boundary value problems in Hilbert spaces. J. Math. Anal. Appl. 303, 736-753 (2005)

16. Manásevich, R., Mawhin, J.: Periodic solutions for nonlinear systems with $p$-Laplacian-like operators. J. Differ. Equ. 145(2), 367-393 (1998)

17. Manásevich, R., Mawhin, J.: The spectrum of $p$-Laplacian systems with various boundary conditions and applications. Adv. Differ. Equ. 5, 1289-1318 (2000)

18. Mugnai, D., Papageorgiou, N.S.: Resonant nonlinear Neumann problems with indefinite weight. Ann. Sc. Norm. Super. Pisa, Cl. Sci. (5) XI, 729-788 (2012)

19. Papageorgiou, N.S., Papalini, F.: Multiple solutions for nonlinear periodic systems with combined nonlinearities and a nonsmooth potential. J. Nonlinear Convex Anal. 13, 681-698 (2012)

20. Papageorgiou, N.S., Winkert, P.: Applied Nonlinear Functional Analysis. de Gruyter, Berlin (2018)

21. Papageorgiou, N.S., Vetro, C., Vetro, F.: Nonlinear multivalued Duffing systems. J. Math. Anal. Appl. 468, 376-390 (2018)

22. Papageorgiou, N.S., Rădulescu, V.D., Repovš, D.D.: Nonlinear Analysis - Theory and Methods. Springer, Switzerland (2019)

23. Papageorgiou, N.S., Rădulescu, V.D., Repovš, D.D.: Relaxation methods for optimal control problems. Bull. Math. Sci. 10, 2050004 (2020)

24. Papageorgiou, N.S., Vetro, C., Vetro, F.: Relaxation for a class of control systems with unilateral constraints. Acta Appl. Math. 167, 99-115 (2020) 
25. Pruszko, T.: Topological degree methods in multivalued boundary value problems. Nonlinear Anal. 9, 959-973 (1981)

26. Zhang, Q., Li, G.: On a class of second order differential inclusions driven by the scalar $p$-Laplacian. Nonlinear Anal. 72, 151-163 (2010)

Publisher's Note Springer Nature remains neutral with regard to jurisdictional claims in published maps and institutional affiliations. 